\title{
Effectiveness of interventions to improve rates of intravenous thrombolysis using behaviour change wheel functions: a systematic review and meta-analysis
}

Md Golam Hasnain ${ }^{1 *}$ (D), John R. Attia ${ }^{1,2,3}$, Shahinoor Akter ${ }^{1,4}$, Tabassum Rahman ${ }^{1,5}$, Alix Hall ${ }^{2}$, Isobel J. Hubbard ${ }^{1}$, Christopher R. Levi ${ }^{1,6}$ and Christine L. Paul ${ }^{1,2}$

\begin{abstract}
Background: Despite being one of the few evidence-based treatments for acute ischemic stroke, intravenous thrombolysis has low implementation rates-mainly due to a narrow therapeutic window and the health system changes required to deliver it within the recommended time. This systematic review and meta-analyses explores the differential effectiveness of intervention strategies aimed at improving the rates of intravenous thrombolysis based on the number and type of behaviour change wheel functions employed.

Method: The following databases were searched: MEDLINE, EMBASE, PsycINFO, CINAHL and SCOPUS. Multiple authors independently completed study selection and extraction of data. The review included studies that investigated the effects of intervention strategies aimed at improving the rates of intravenous thrombolysis and/or onset-to-needle, onset-to-door and door-to-needle time for thrombolysis in patients with acute ischemic stroke. Interventions were coded according to the behaviour change wheel nomenclature. Study quality was assessed using the QualSyst scoring system for quantitative research methodologies. Random effects meta-analyses were used to examine effectiveness of interventions based on the behaviour change wheel model in improving rates of thrombolysis, while meta-regression was used to examine the association between the number of behaviour change wheel intervention strategies and intervention effectiveness.

Results: Results from 77 studies were included. Five behaviour change wheel interventions, 'Education', 'Persuasion', 'Training', 'Environmental restructuring' and 'Enablement', were found to be employed among the included studies. Effects were similar across all intervention approaches regardless of type or number of behaviour change wheelbased strategies employed. High heterogeneity $\left(I^{2}>75 \%\right)$ was observed for all the pooled analyses. Publication bias was also identified.

Conclusion: There was no evidence for preferring one type of behaviour change intervention strategy, nor for including multiple strategies in improving thrombolysis rates. However, the study results should be interpreted with caution, as they display high heterogeneity and publication bias.
\end{abstract}

Keywords: Thrombolysis, Implementation, Intervention, Systematic review, Meta-analysis

\footnotetext{
* Correspondence: mdgolam.hasnain@uon.edu.au

'School of Medicine and Public Health (SMPH), University of Newcastle (UoN), Callaghan, New South Wales, Australia

Full list of author information is available at the end of the article
}

(c) The Author(s). 2020 Open Access This article is licensed under a Creative Commons Attribution 4.0 International License, which permits use, sharing, adaptation, distribution and reproduction in any medium or format, as long as you give appropriate credit to the original author(s) and the source, provide a link to the Creative Commons licence, and indicate if changes were made. The images or other third party material in this article are included in the article's Creative Commons licence, unless indicated otherwise in a credit line to the material. If material is not included in the article's Creative Commons licence and your intended use is not permitted by statutory regulation or exceeds the permitted use, you will need to obtain permission directly from the copyright holder. To view a copy of this licence, visit http://creativecommons.org/licenses/by/4.0/. The Creative Commons Public Domain Dedication waiver (http://creativecommons.org/publicdomain/zero/1.0/) applies to the data made available in this article, unless otherwise stated in a credit line to the data. 


\section{Contribution to the literature}

This study is the first rigorous systematic review and metaanalysis, which evaluates the differential effect of intervention strategies aimed at improving rates of intravenous thrombolysis based on behaviour change wheel intervention function as an analytical framework.

This review illustrates that this field of research not only has high heterogeneity as previously known, but also provides new evidence of publication bias both within and across intervention types.

Although this study indicates that various strategies can be effective, it does not provide strong evidence supporting any specific strategy. Most studies do not have enough detail to unambiguously classify the intervention components.

\section{Background}

Stroke causes 5.5 million deaths worldwide and requires substantial treatment and post-stroke care-related economic costs [1]. There are an estimated 80 million stroke survivors worldwide, with an increase in absolute numbers of disability-adjusted life years [1]. Acute ischemic stroke (AIS) refers to the most prevalent and disabling form of stroke [2]. Intravenous thrombolysis (IVT) is considered one of the mainstream therapies for AIS since its approval in 1996 by the United States Food and Drug Administration as a first-line treatment [3]. Despite substantial evidence for both the safety and costeffectiveness of IVT, the implementation rate has remained persistently low [4]. Subsequently, over 50 published studies deploying a variety of trial designs have tested a variety of approaches to boost implementation rates. One major challenge for increasing IVT usage is reducing onset-to-needle time, the sum of the onsetto-door and the door-to-needle times. Several strategies to reduce door-to-needle time have already been tested and have achieved improvements in IVT rates. Delayed patient arrival at hospital remains, however, one of the major obstacles to better IVT implementation with many studies focusing on approaches aiming to reduce pre-hospital delay [5]. Several additional factors, relevant at both individual and organisational levels, have been identified as major rate-limiting factors for IVT implementation [6]. Patients' and bystanders' inability to recognise stroke symptoms and signs resulting in delayed response in seeking support from healthcare providers contributes to delayed hospital arrival [6]. Delays in stroke recognition by paramedics and hospital staff, delays in obtaining and interpreting brain imaging, inefficiencies in emergency stroke care, delays in obtaining treatment consent, an absence of decision support systems and protocols in emergency care facilities and physician perception of IVT efficacy and safety have also been identified as major factors that limit IVT implementation [5]. Consequently, several, often multi-faceted intervention strategies have been tested in efforts to improve the rates of IVT in AIS [7]. Such intervention strategies include telemedicine and 'hub-spoke' models, pre- and/or in-hospital notification, multi-disciplinary collaborative approaches and re-organisation of prehospital and hospital systems of care $[4,7]$.

Since the intervention strategies to date have used a variety of methods in various settings, we aim to perform a systematic review and meta-analysis to compare the effectiveness of the various forms of intervention strategies. Thus far, there are three published systematic reviews that have attempted to investigate these issues; however, each has limitations. The first systematic review, published in 2016, only included studies that met the Cochrane collaboration standards for practice and organisation of care study design criteria [8]. The second and third systematic reviews and meta-analyses were published in 2018 and 2019 [4, 7]. Both studies expressed the meta-analyses results based on various intervention approaches, but they did not use any specific operational definitions or theoretical approaches when grouping the studies in the analyses [4, 7]. Moreover, neither study explored publication bias when describing group-based results [4, 7], despite the importance of this information to data interpretation.

Theory-based analysis of interventions is recommended when investigating the effect of a specific intervention strategy and aiding in the specification of a potentially active process of care [9]. For example, behaviour-targeted theories can be utilised to define the components of implementation interventions [10]. Multi-level, multi-disciplinary testing and decisionmaking processes are needed to identify a patient's eligibility for IVT [11]. Therefore, increasing rates of IVT in AIS are considered an example where multiple factors could be critical to the design of targeted intervention strategies [8]. Conceptual frameworks, such as the behaviour change wheel (BCW), can be useful in defining the range of factors (e.g. training) that need to be addressed to effect complex change [12]. BCW is a behavioural framework which has at its centre the Capability, Opportunity, Motivation-Behaviour (COM-B) theory. As described by Nilsen et al. 2015 [13], the COM-B is an implementation theory which is useful for providing an understanding or an explanation of aspects of implementation. The overarching BCW framework specifies intervention functions (e.g. education, persuasion, training), which can be used to develop intervention content (i.e. a process model) and guide evaluation of an implementation intervention (e.g. an evaluation framework) [13]. Therefore, the BCW framework provides a useful 
Table 1 Operational definitions for the assessed intervention components

\section{Intervention component}

Education

Persuasion

Training

Environmental Restructuring

Enablement

\section{Definition}

Providing systematic education or instruction to increase knowledge or understanding on stroke via face-to-face or online educational session, or by providing print or online educational materials to the health professionals from any level or community members.

Improve communication to stimulate the treatment process in patients with stroke.

Providing systematic training to the community members or health professionals from any level to improve their skills in identifying suspected stroke cases.

AND OR

Providing systematic training to the health professionals from any level to improve their skills in diagnosing and treating stroke cases.

Restructuring, reorganising or rearranging individual, social or organisational context to promote the usage of thrombolysis in stroke.

Increasing resources such or reducing obstacles to increase capability or opportunity at the individual level, e.g. health care staff or organisational level, e.g. hospital to promote the process and quality of stroke care.

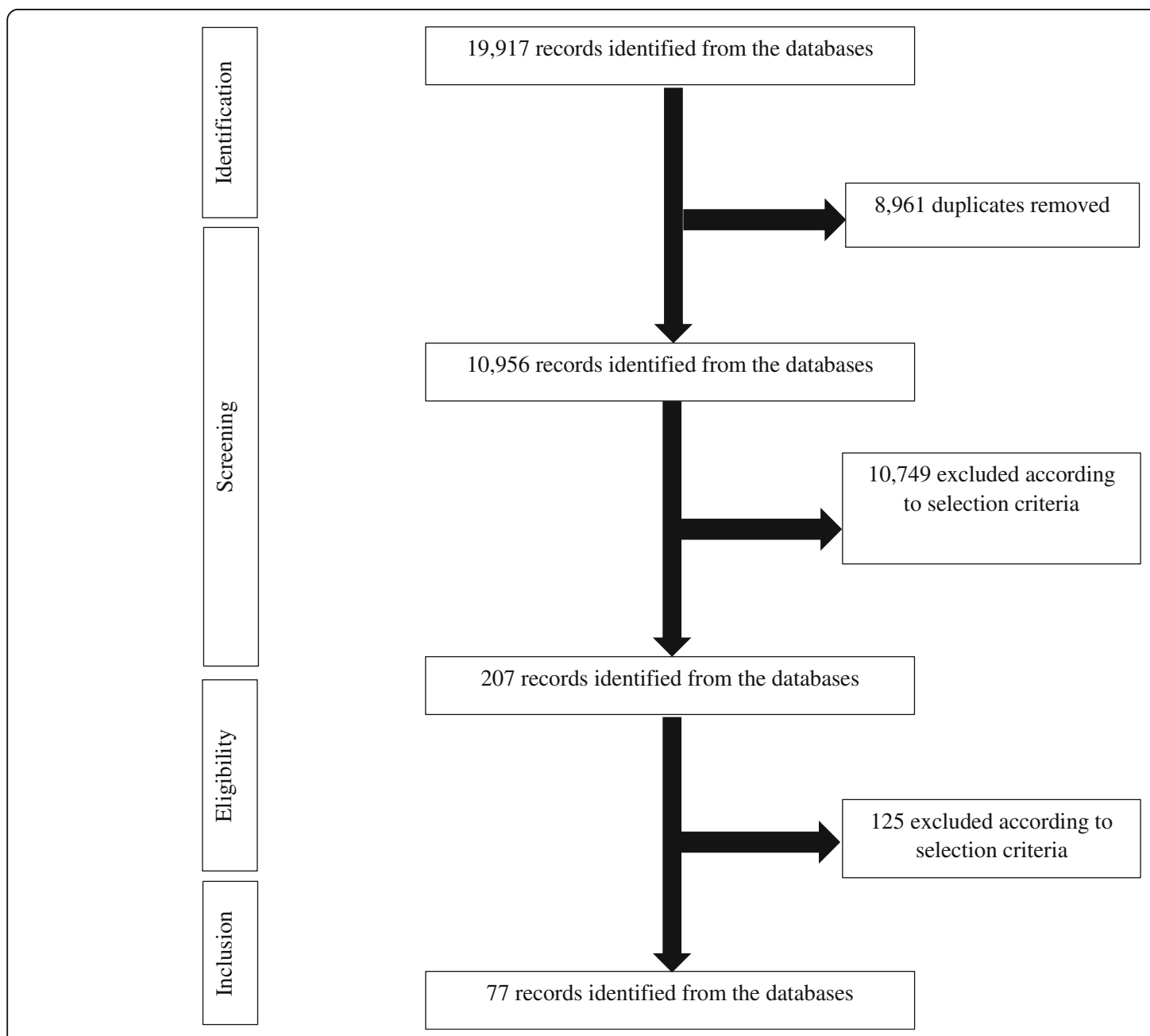

Fig. 1 Flow diagram of studies included in the systematic review 
Hasnain et al. Implementation Science

(2020) $15: 98$

Page 4 of 21

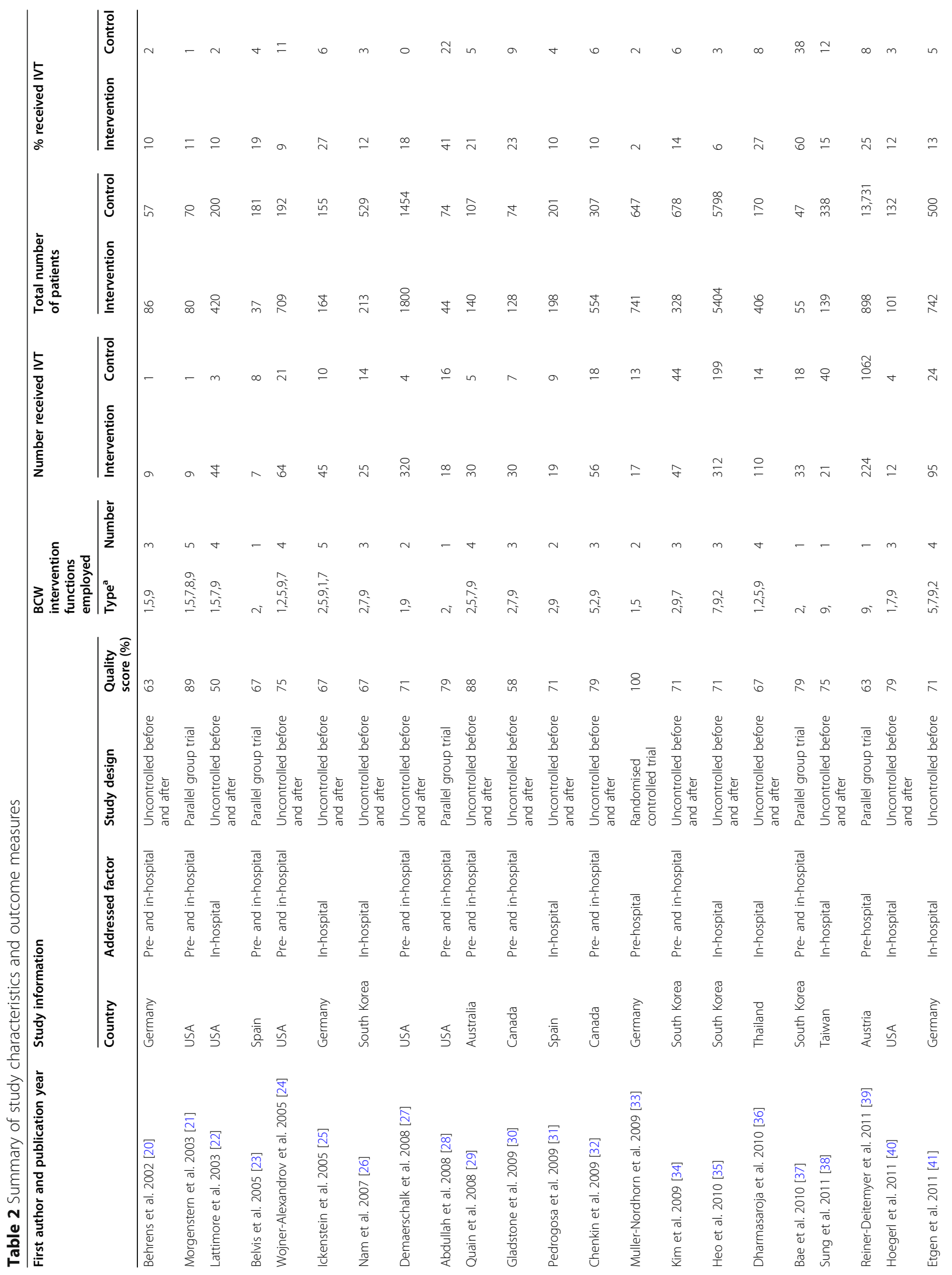


Hasnain et al. Implementation Science $\quad$ (2020) 15:98

Page 5 of 21

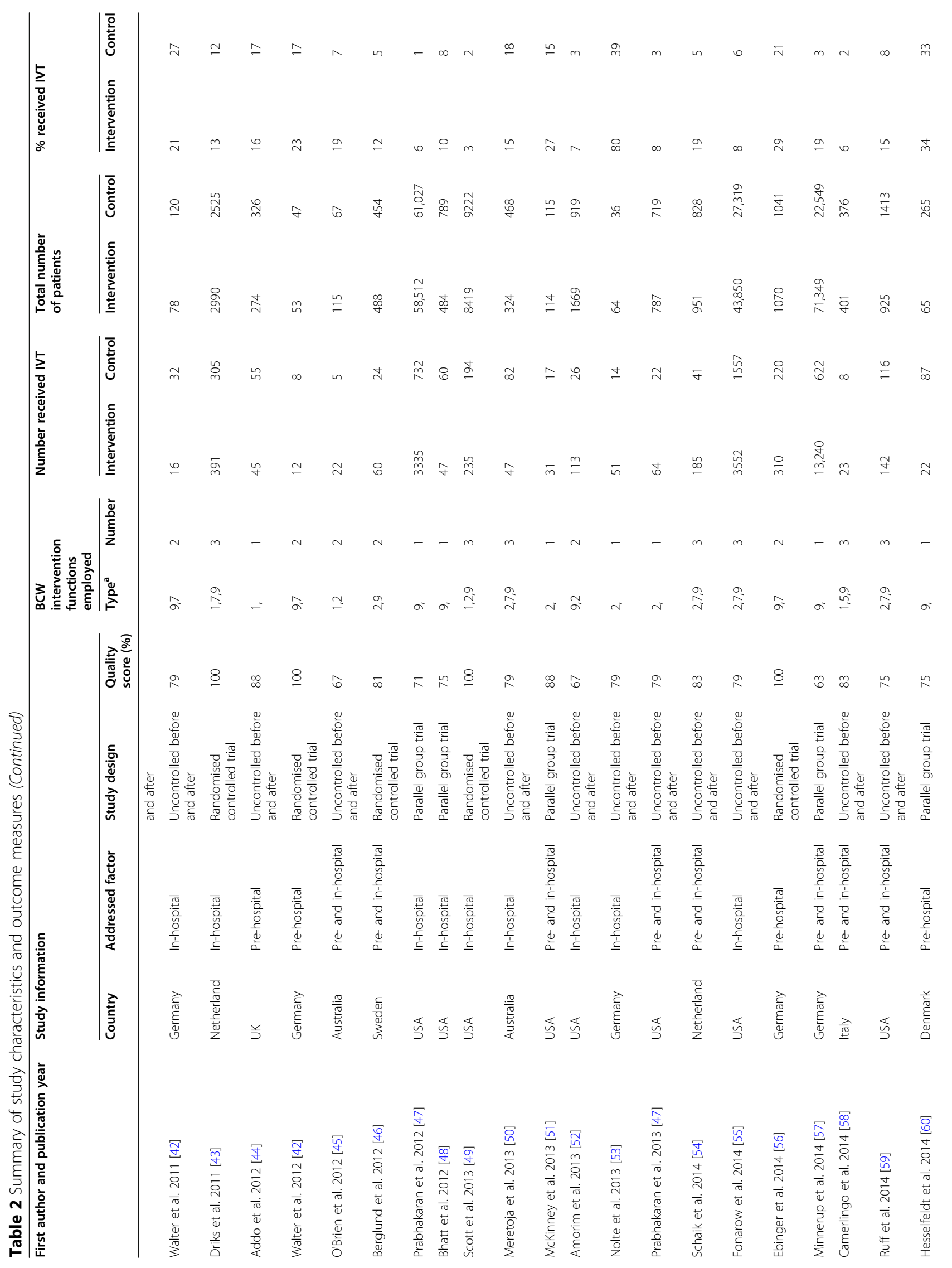




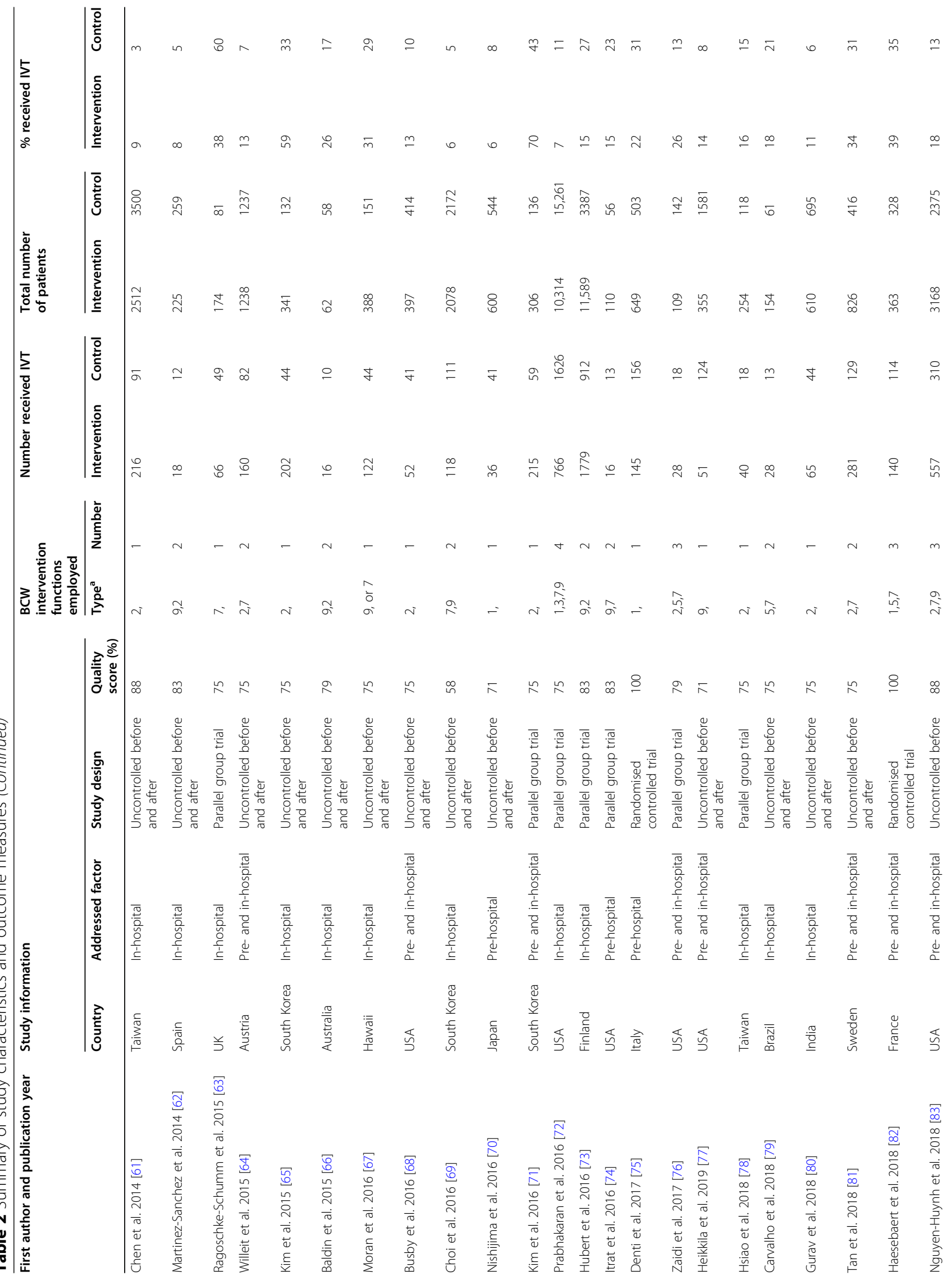




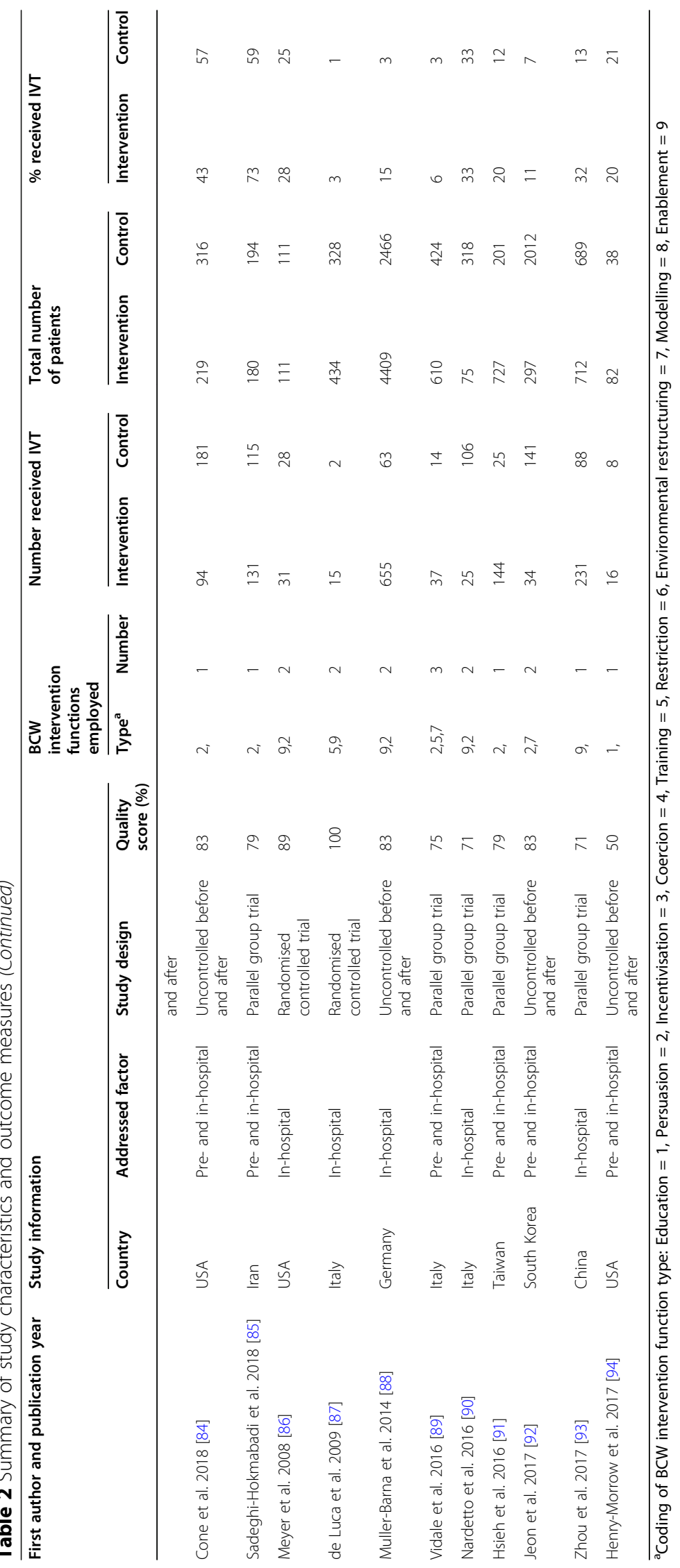


structure for categorising and understanding the content of previous interventions and considering their potential implications; particularly in the context of literature where in-depth detail about intervention content is not commonly provided. In the context of stroke care, considering the existing literature in terms of intervention functions rather than solely pooling all implementation studies has the potential to provide additional understanding regarding how to successfully implement a complex multi-component practice like IVT in a given healthcare organisation. Thus, an evaluation of the interventions aimed at improving the rates of IVT in AIS including coding them based on $\mathrm{BCW}$ intervention function could assist to identify more clearly the approaches which might be associated with higher rates of IVT implementation.

The core aspect of the $\mathrm{BCW}$ framework described by Michie et al. consists of a 'behaviour system' that includes three key elements: capability, opportunity and motivation. This core aspect is surrounded by nine intervention functions and then by seven policy categories [12]. The intervention functions help to identify the gaps and highlight the areas that need intervention. For example, the intervention functions were used to characterise interventions related to smoking cessation and reducing obesity [12]. The functions can also be used to contextualise already implemented interventions and to lead to more efficient design of effective interventions.

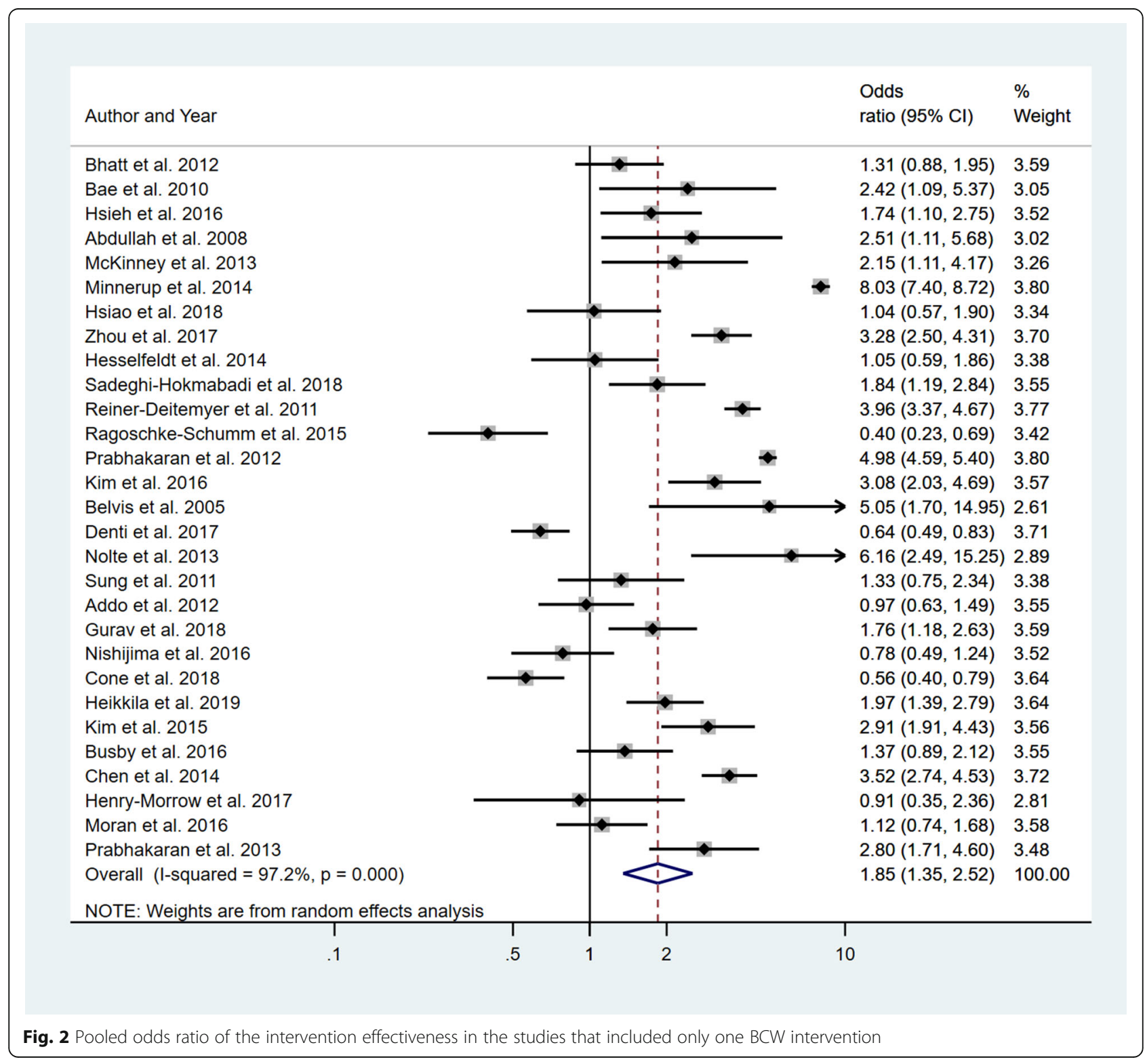


To date, no studies have used the BCW classification as a framework for examining the intervention strategies aimed at improving the rates of IVT. To synthesise the results of studies which tested the effect of intervention strategies aimed at improving the rates of IVT in AIS, we will use the $\mathrm{BCW}$ nomenclature as the analytical context with the following primary and secondary objectives:

* Primary objective: to explore the differential effectiveness of the intervention strategies aimed at improving the rates of IVT based on the number and type of $\mathrm{BCW}$ intervention functions employed.

* Secondary objective: to describe the number and type of $\mathrm{BCW}$ intervention functions employed in intervention strategies aimed at improving the rates of IVT.

\section{Methods}

This systematic review follows the Preferred Reporting Items for Systematic reviews and Meta-analyses (PRIS MA) statement guidelines [14]. The PRISMA statement is provided in Supplementary file 1. This systematic review was not registered.

\section{Searches}

MEDLINE, EMBASE, PsycINFO, CINAHL and SCOPUS databases were searched for articles published from January 1996 to December 2018 in English. We also checked the reference lists of included articles and existing systematic reviews for relevant studies. The search dates were selected to coincide with the 1996 approval and release of the first thrombolysis guideline for AIS [15].

We followed the search strategy described by Paul et al. 2016 [8] while selecting the search terms. The search terms are a combination of keyword searches:

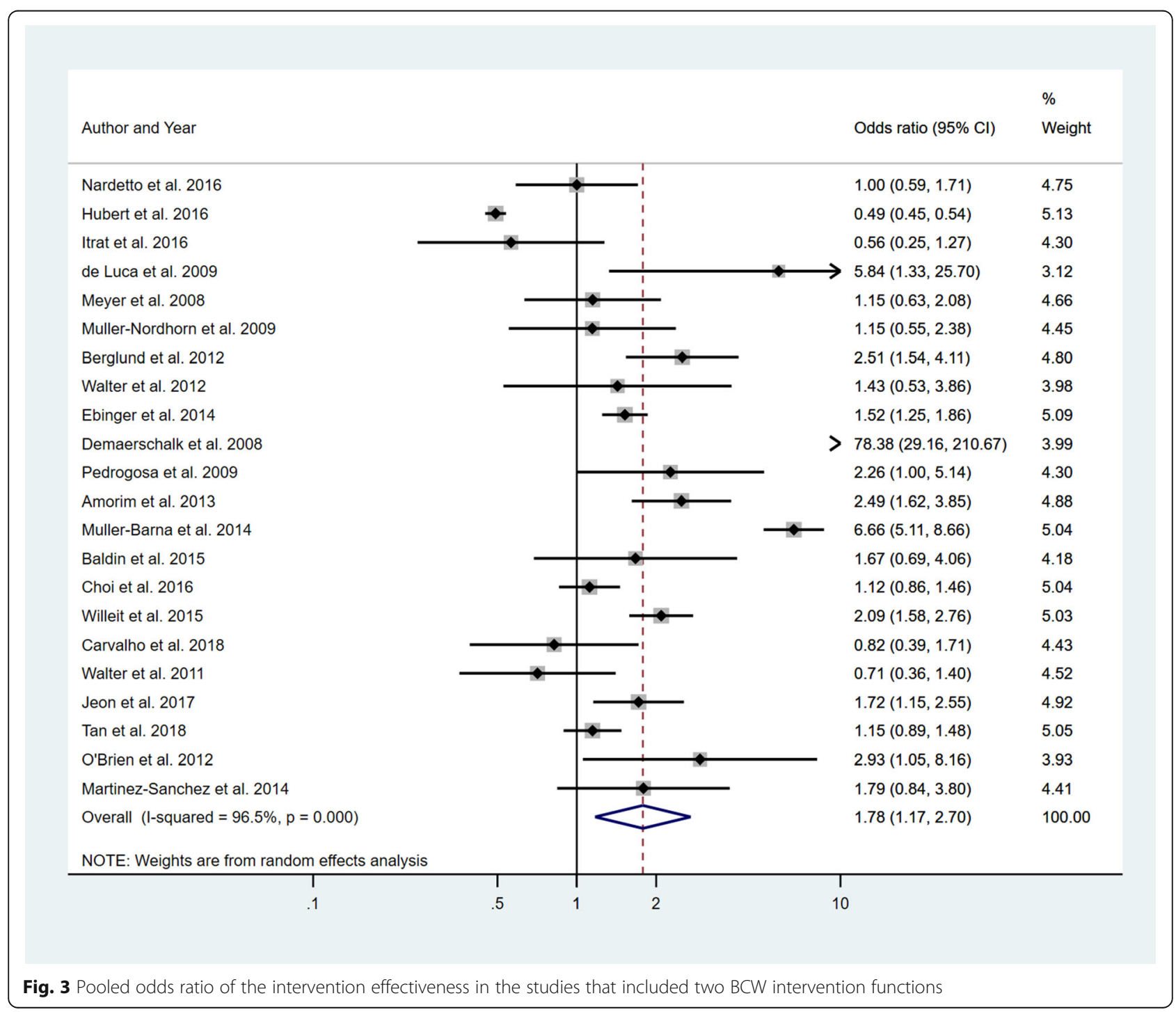


'Tissue plasminogen activator' OR 'tPA' OR 'rtPA' OR 'Alteplase' OR 'Thrombolysis' AND 'Stroke' OR 'Ischemic stroke' OR 'Brain ischemia' OR 'Middle cerebral artery infarction' OR 'Cerebrovascular disorder' OR 'Cerebrovascular accident' OR 'CVA' OR 'Cerebral stroke' OR 'Cerebral accident' OR 'Cerebral infarction' OR 'Cerebral apoplexy' OR 'Cerebrovascular apoplexy'. We used available MeSH headings; otherwise, a 'multipurpose; mp.' field search was conducted. One senior librarian reviewed the final search strategy. The detailed search strategy is provided in Supplementary file 2.

\section{Inclusion and exclusion criteria}

This systematic review included the following: quantitative studies that investigated the effect of interventions to improve the rates of IVT and/or onset-to-needle, onset-to-door and door-to-needle time for IVT in patients with AIS; studies that reported the rates of IVT utilisation and/or onset-to-door and/or door-to-needle time for thrombolysis as their primary outcome; studies that reported the number of patients with AIS who received IVT and the total number of suspected stroke and/or confirmed stroke and/or confirmed ischemic stroke patients and/or the total number of eligible patients for IVT; randomised controlled trials and clusterrandomised trials; non-randomised studies, such as uncontrolled before-after studies; parallel group trials; and observational studies including cohort, case-control and cross-sectional studies. The systematic review included a wide range of study designs to identify the possible reasons and factors behind the systematic review result [16]. Inclusion was also limited to original human research studies.

The review excluded studies that reported only haemorrhagic stroke or transient ischemic attack and those reporting rates of thrombolysis other than IVT. Key exclusion and inclusion points are summarised in Supplementary file 3 .

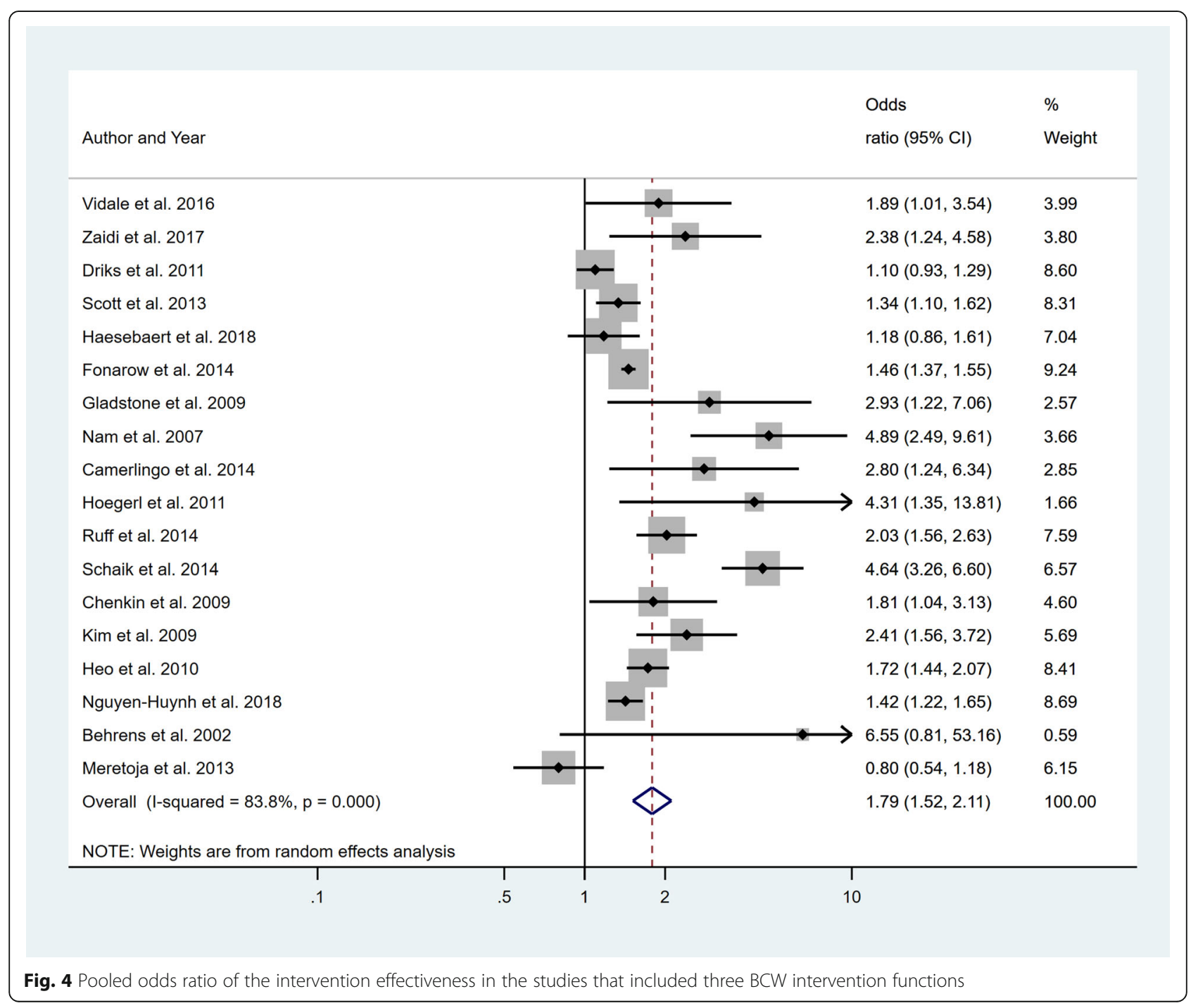




\section{Outcome measures}

We used IVT rates as our outcome of interest. The numerator was the total number of patients who received IVT, and the denominator was the total number of suspected stroke, confirmed stroke, confirmed ischemic stroke or IVT-eligible patients.

\section{Potential effect modifiers and reasons for heterogeneity}

Outcome measures were grouped as follows, to explain some of the heterogeneity across the studies:

* Hospital factors addressed by the intervention (prehospital, in-hospital or both pre- and in-hospital)

* Denominator used (suspected stroke, confirmed stroke, confirmed ischemic stroke or IVT-eligible patients).

* Epidemiological design of the study (uncontrolled before-and-after, parallel group trial or randomised controlled trial).

The number of $\mathrm{BCW}$ intervention functions employed was included as a covariate in a meta-regression to examine the association between number of $\mathrm{BCW}$ intervention functions employed and intervention effectiveness and to explain the heterogeneity between the studies.

\section{Study quality assessment}

Three reviewers assessed the quality of the studies. The principal reviewer (MGH) independently assessed the methodological quality of the final articles using the QualSyst scoring system for quantitative research methodologies [17]. The total number of included studies was then divided between two independent reviewers (SA and TR), who also assessed the quality of the studies using the same scoring system. Joint discussion between all three reviewers resolved any disagreements. Studies were scored depending on whether they fully met the criteria ( 2 points), partially met the criteria (1 point) or did not meet the criteria at all ( 0 points). Quantitative studies were scored against 14 criteria. A criterion for 'evidence of ethical approach' was added to the QualSyst scoring, resulting in a maximum total possible score of 22 for qualitative designs and 30 for quantitative designs [18].

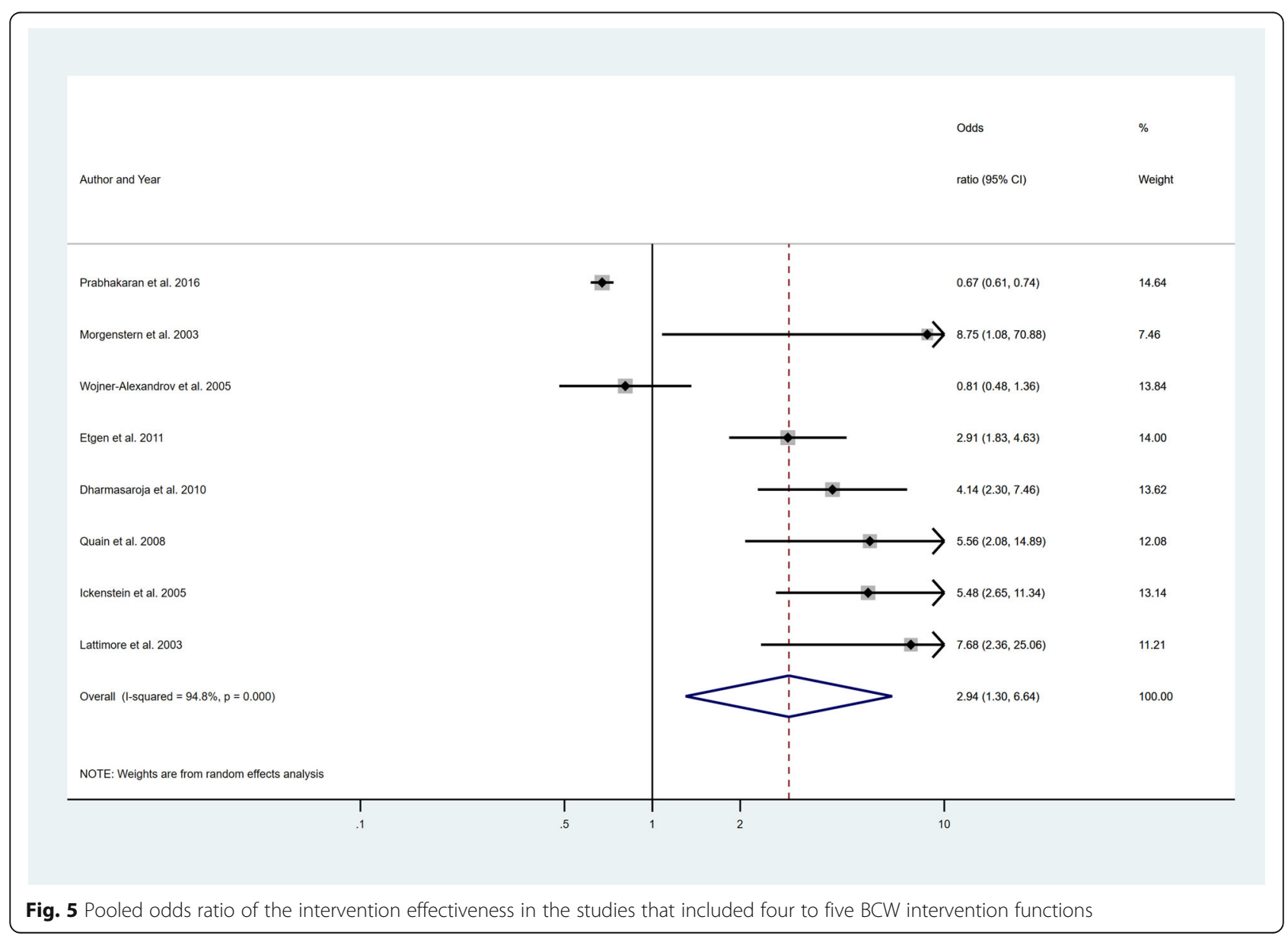




\section{Study selection}

All reviewers reviewed the titles and abstracts of the last 500 search results to identify abstracts that would potentially meet the inclusion criteria. There was $>95 \%$ agreement between reviewers. After removing duplicates, the principal reviewer reviewed all titles and abstracts and the full text of each non-rejected article to arrive at final inclusion determinations. The other two reviewers concurrently and independently reviewed half of the titles and abstracts and the full texts of non-rejected articles each and then compared their determinations to those made by the principal reviewer. The study selection process is described in more detail in Supplementary file 4 and Supplementary file 5

\section{Data extraction and coding strategy}

The principal reviewer performed data extraction for all articles independently identified as 'included'. A data extraction form was developed following the format used in the Cochrane Handbook for Systemic Reviews of Interventions [19]. This process recorded the following information: country, setting, publication year, intervention duration, study type based on epidemiological design, description of the intervention and outcome. The outcome used the total number of patients receiving IVT as the numerator and the total number of suspected stroke, confirmed stroke, confirmed ischemic stroke or IVTeligible patients as the denominator. To ensure consistency, a data extraction form was pilot-tested on a $5 \%$ subset of the included full-text studies.

The interventions in the included studies were coded according to $\mathrm{BCW}$ framework intervention functions criteria mentioned in Michie et al [10].. Operational definitions for the included intervention functions have been described in Table 1. Three reviewers were involved in the process of coding. The principal author (MGH) independently identified the BCW intervention functions addressed through the interventions of the 77 studies. The

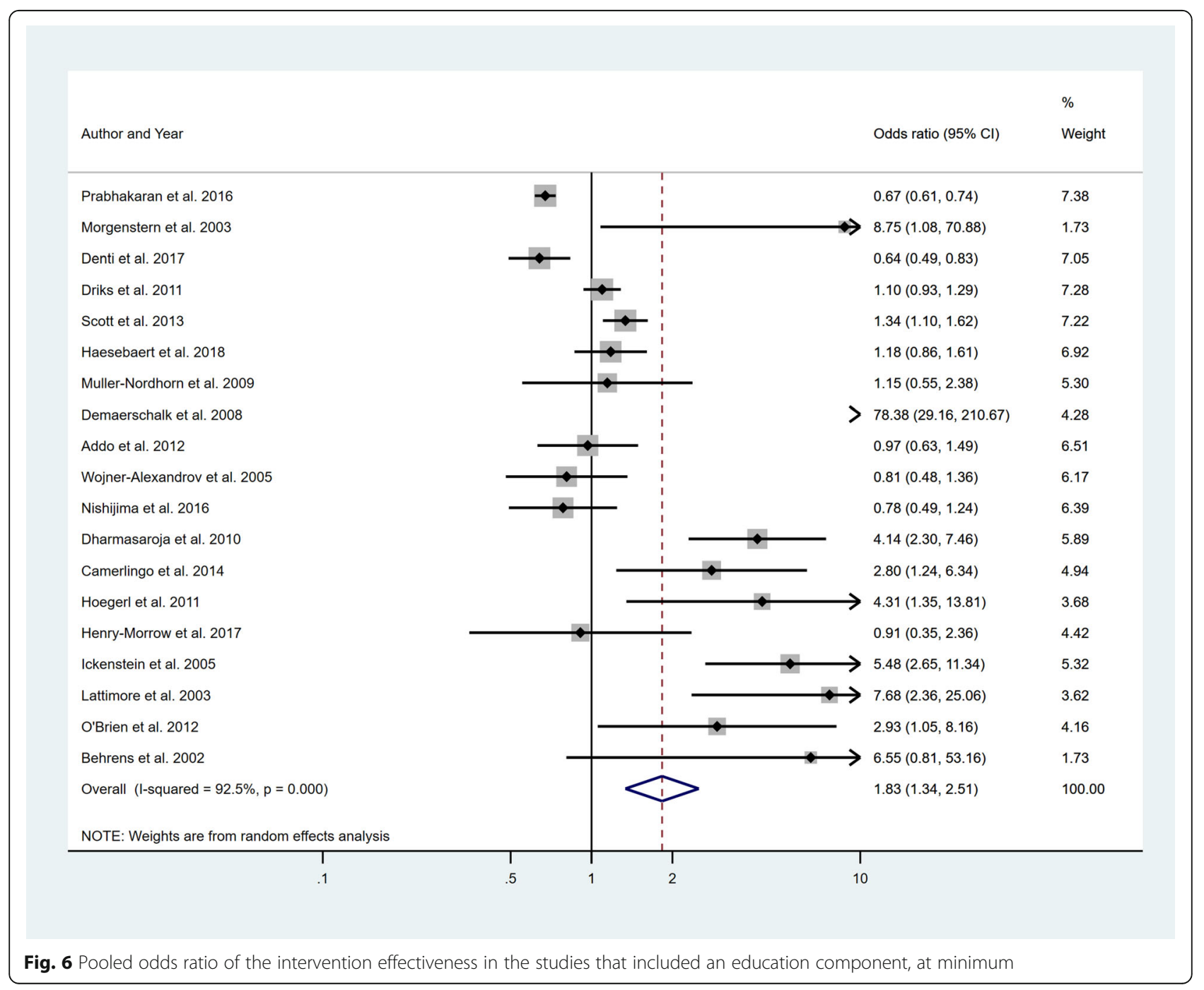


total number of included studies was then divided between another two co-authors (SA and TR), who also identified the $\mathrm{BCW}$ intervention functions addressed through the interventions of the 38 and 39 studies respectively. The discrepancy between MGH and SA was $10.5 \%$ (4 out of 38 ) and between MGH and TR was $7.7 \%$ (3 out of 39). Joint discussion between all three reviewers resolved any disagreements. All coding discrepancies were then reviewed and finalised by the senior author $(\mathrm{CP})$.
Studies were categorised based on the number and type of BCW interventions employed. Specifically, studies were categorised based on:

* The number of BCW components implemented as part of the intervention, with categories including 1 , 2,3 or $>3$.

* Whether one of the following components were included as part of the intervention: education,

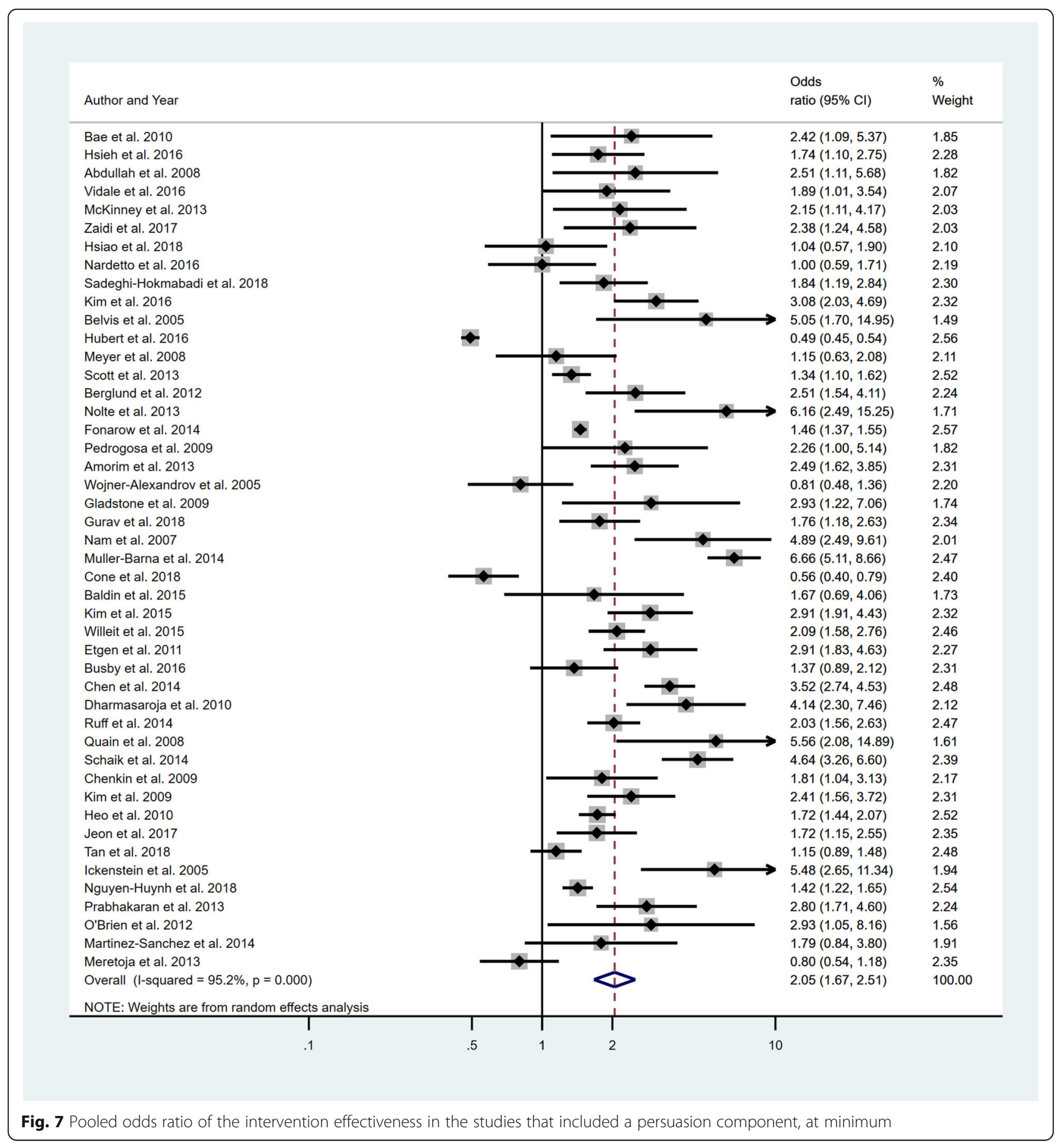


persuasion, incentivisation, coercion, training, restriction, environmental restructuring, modelling and enablement.

\section{Statistical analyses}

Separate random effects meta-analyses by type of $\mathrm{BCW}$ strategy (i.e. education, persuasion, training, environmental, restructuring and enablement) and by number of $\mathrm{BCW}$ strategies implemented (i.e. one, two, three and more than 3 ) were conducted to assess the effectiveness of the interventions on improving IVT rates (primary objective). For each meta-analysis, a pooled odds ratio (OR) with a $95 \%$ confidence interval $(\mathrm{CI})$ was calculated using the metan command in Stata. In addition, forest plots were used to show the effect sizes, to assess possible heterogeneity and identify potential outliers. Statistical heterogeneity was assessed by chi-square and $I^{2}$ statistics. Publication bias was assessed by testing the asymmetry of funnel and contour-enhanced funnel plots, which were created using the metafunnel and confunnel commands in Stata, and via Egger's test, which was conducted using the metabias command which was used in Stata. The impact of potential outliers on the results was assessed by conducting sensitivity analyses whereby any identified outliers were removed and the analysis rerun. Descriptive analyses were used to report the number and type of $\mathrm{BCW}$ framework interventions used as part of the study intervention (secondary objective). Finally, to determine if the number of $\mathrm{BCW}$ intervention strategies used were associated with intervention effectiveness, meta-regression was conducted using the metareg command in Stata. Stata (StataCorp LLC, College Station, Texas, USA) version 14 was used to conduct all analyses.

\section{Results}

\section{Description of studies}

A total of 19,917 titles were screened following database searches and hand searching of bibliographies, and 8961 titles were excluded (Fig. 1). The search hits of all databases are showed in Supplement 2. The remaining 10,749 abstracts were reviewed. A total of 207 articles were included

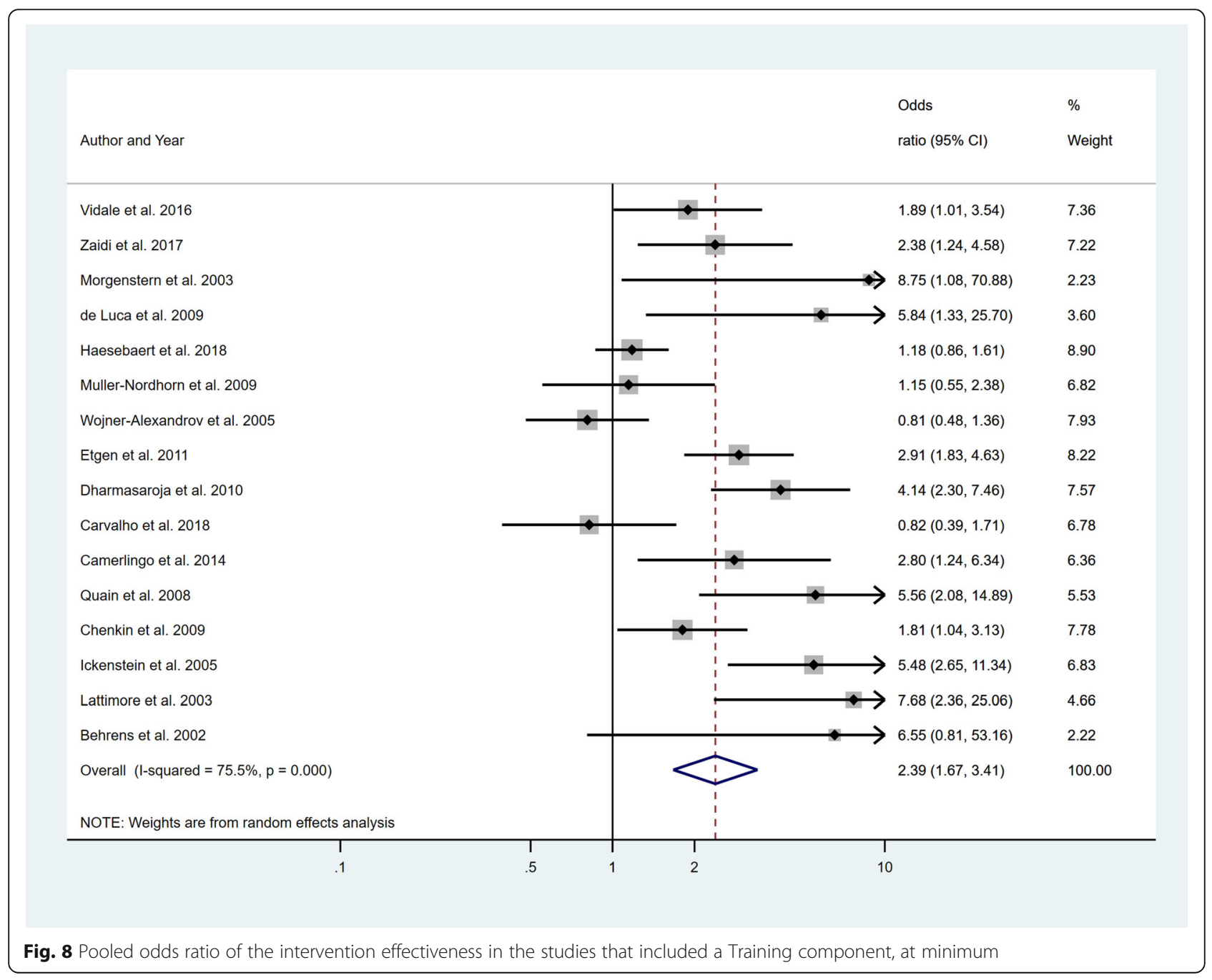


for full-text data review, and 77 were selected based on the inclusion and exclusion criteria. BCW classifications were coded for all interventions described in the eligible manuscripts and then included in the meta-analyses.

\section{Characteristics of studies}

The 77 studies included in the meta-analyses represent 40,614 IVT cases, and their general characteristics are shown in Table 1. All were published between 2002 and 2018. There were 29 (38\%) studies from European countries, 26 (34\%) from North American countries, 17 (22\%) from Asian countries, four (5\%) from Australia and one (1\%) from South America. In the methods used, 58\% (45/77) were uncontrolled before-and-after, 29\% (22/77) were parallel group trial and 13\% (10/77) were randomised controlled in design. For the factors the interventions addressed, 35 (45\%) addressed in-hospital factors, 33 (43\%) addressed pre- and in-hospital factors and nine $(12 \%)$ addressed pre-hospital factors only.

\section{Quality of studies}

The quality scores across studies were normally distributed with a mean of 78 and SD of 11, as shown in Table 2. The highest mean score of 97 (SD 7) was observed in the randomised controlled trial group, followed by similar scores of 76 (SD 7) and 74 (SD 9) for the parallel group trial and the uncontrolled before-and-after study group, respectively.

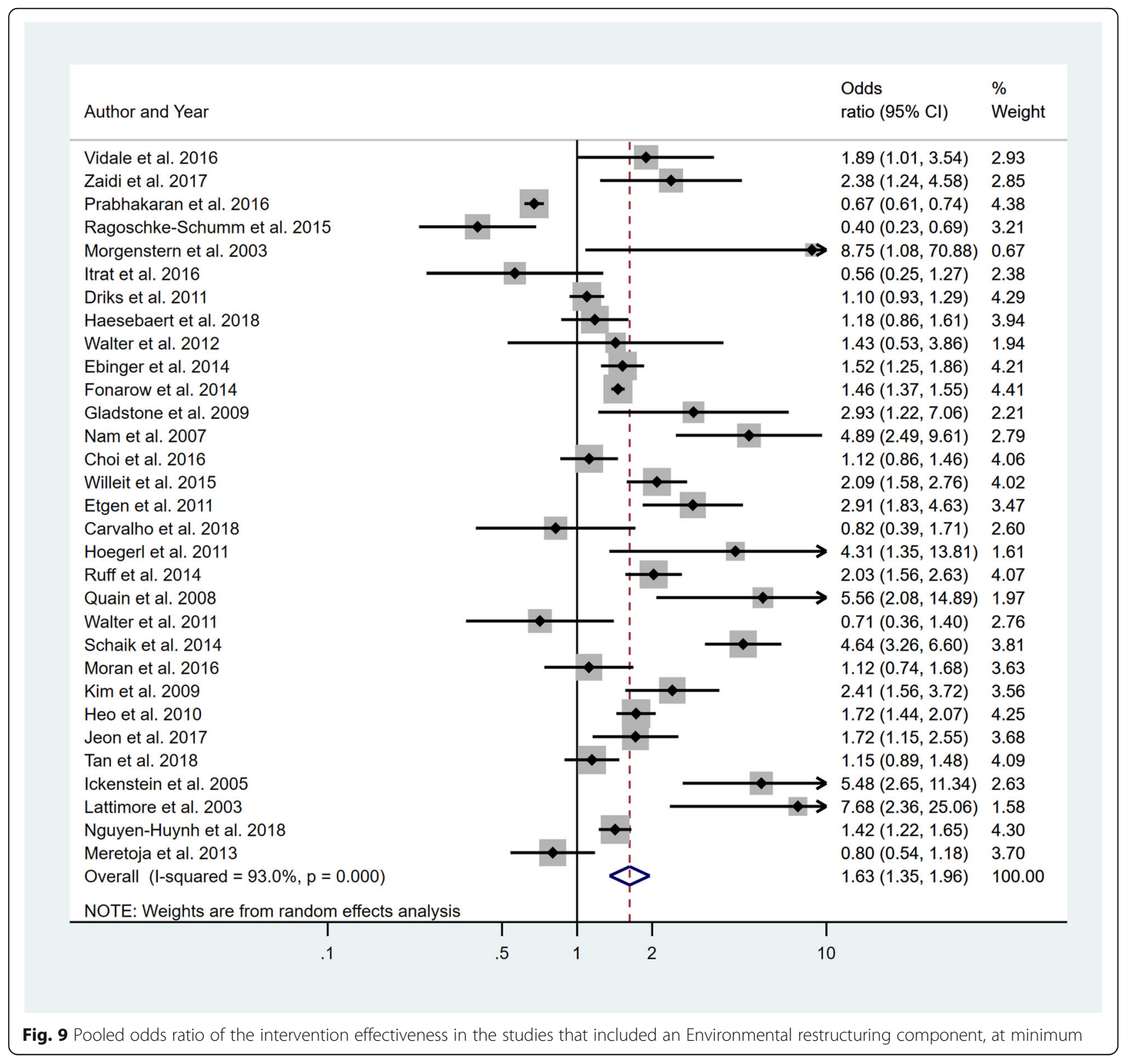




\section{Description on behaviour change wheel categories}

Twenty nine (38\%) studies implemented one BCW component, 22 (29\%) implemented two components, 18 (23\%) implemented three components and $8(10 \%) \mathrm{im}$ plemented more than 3 components. Of the types of BCW interventions included, 47 (61\%) studies included at least enablement, $46(60 \%)$ included at least persuasion, $31(40 \%)$ at least environmental restructuring, 19 $(25 \%)$ at least education and $16(21 \%)$ at least training.

\section{Outcome measures}

Four separate meta-analyses were conducted to assess the effect of incorporating one, two, three or more $\mathrm{BCW}$ intervention types (Fig. 2, 3, 4 and 5). All four analyses found significant overall improvements in rates of IVT delivery, with odds ratio of between 1.78 to 2.94, with largely overlapping confidence intervals. High heterogeneity was seen in all meta-analyses ( $I^{2}$ range 84 to $97 \%$ ). Sub-group analyses based on the hospital factors

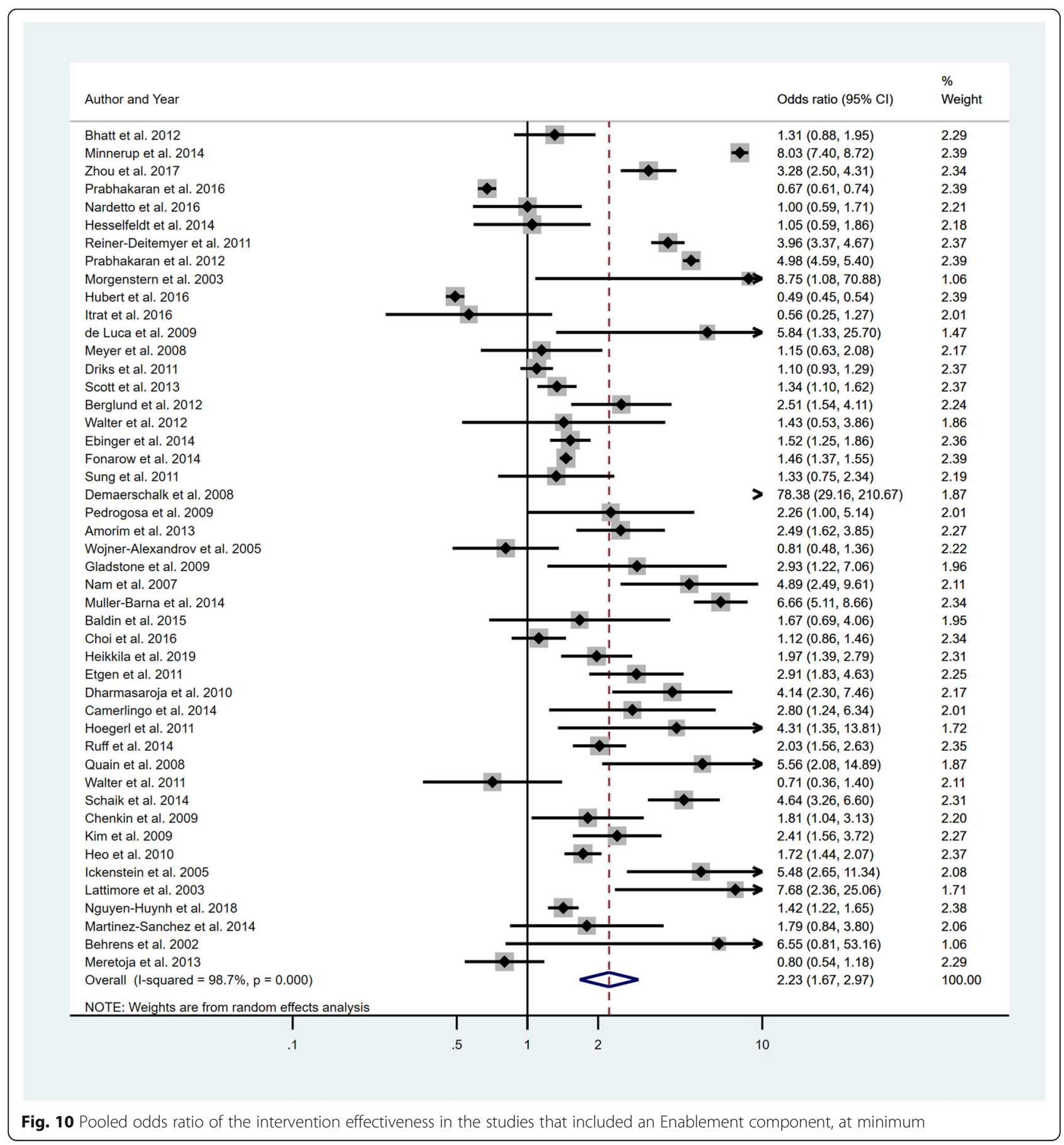


addressed by the intervention, the denominator used and epidemiological design of the study indicated that heterogeneity $\left(I^{2}\right)$ still ranged from moderate to high. A sensitivity analysis that excluded the results of an outlier study (Demaerschalk et al. [27]) did not result in any substantial change in the results or conclusions.

Five separate meta-analyses (Figs. 6, 7, 8, 9 and 10) were conducted to assess the effect of including at least one of the five $\mathrm{BCW}$ intervention types. All five found significant improvement in rates of IVT delivery, with odds ratios of between 1.63 and 2.39 with largely overlapping confidence intervals. High heterogeneity was seen in all meta-analyses ( $I^{2}$ range 75.5 to $\left.98.8 \%\right)$. Sub-group analyses based on hospital factors addressed by the intervention, denominator used and epidemiological design of the study were again conducted and exhibited moderate to high levels of heterogeneity $\left(I^{2}\right)$. A sensitivity analysis excluding the results of the outlier, Demaerschalk et al. [27] study, was again performed, but this did not substantially change the results.

The meta-regression analysis undertaken to assess whether the number of $\mathrm{BCW}$ interventions were associated with efficacy, showed no statistically significant association (OR 1.10; 95\% CI 0.93, 1.32, $p=0.26$ ).

The contour-enhanced funnel plots investigating the type and number of $\mathrm{BCW}$ interventions employed indicated the likelihood of a small-study bias across all meta-analyses, and this is confirmed by Egger's test (Supplementary file 6, and Supplementary file 7). The missing regions in the contour-enhanced funnel plots indicate that the bias is likely due to a mix of heterogeneity and publication bias. We analysed our results based on various study types (including pre-hospital versus in-hospital) and evaluated the effect on the outcomes. However, the result was the same-an overall significant result with all sub-groups. This result also indicated high heterogeneity and potential publication bias (Supplementary file 8, Supplementary file 9, and Supplementary file 10). Since RCTs provide the strongest evidence, we also looked at the results within the RCT sub-group. In this sub-group, meta-analysis revealed a statistically significant improvement in promoting IVT delivery with an OR of 1.55 (95\% CI 1.02-2.35) for the interventions including at least persuasion, OR 1.44 (95\% CI 1.04-1.80) for enablement and OR 1.26 (95\% CI 1.031.54) for environmental restructuring.

\section{Discussion}

The findings of this systematic review and meta-analyses bring together empirical evidence regarding the strategies that are potentially effective in improving IVT rates for AIS. This is the first review to evaluate the differential effect of implementation interventions using a theory-informed (i.e. COM-B) approach using the $\mathrm{BCW}$ framework to increase the information available about effective intervrention content. This study found similar overall benefits for the five $\mathrm{BCW}$ intervention approaches reviewed. Using the $\mathrm{BCW}$ nomenclature, education, persuasion, training, environmental restructuring and enablement all increase thrombolysis uptake with odds ratios of approximately 2 . Because the pooled effect sizes were largely overlapping, there seems to be no clear 'winner' in terms of which strategy has the largest effect for improving rates of IVT for AIS.

The COM-B theory proposes that multiple aspects of behaviour (capability, opportunity and motivation) are necessary to performing a given behaviour, and the $\mathrm{BCW}$ framework proposes multiple factors (education, training, etc.) which support and drive behaviour at an individual and system level. Despite the theory-driven expectation that multiple implementation intervention strategies may potentially increase success in the context of a complex multi-step health care practice, we found no evidence that increasing the number of $\mathrm{BCW}$ strategies used in an intervention programme resulted in increased effectiveness. However, the sub-group analysis of RCTs provides some suggestion that persuasion, environmental restructuring and enablement may be particularly effective.

The results should be interpreted with caution based on the existence of both high study heterogeneity or variability of studies, and publication bias. Heterogeneity describes the degree of variability among studies. The presence of high heterogeneity in this study indicates that the true intervention effect may be different in different studies. On the other hand, the presence of bias indicates the possibility of having overestimated the summary effect size [19]. Therefore, until there is more substantial evidence, rather than selecting an intervention approach based on the literature, it may be more relevant to select approaches which best address the major barriers in a given health service context. The findings of the current study align with those of McDermott et al. [4] who concluded that it was difficult to identify any one particular most effective strategy because the overall effects of the identified strategies were almost the same [4]. Conversely, Huang et al. [7] concluded that interventions reducing in-hospital delays may serve as the most effective way to increase IVT delivery; however, several approaches were included under the 'in-hospital' approach, which made it difficult to recommend any specific approaches, and they did not find strong evidence to suggest a minimum or maximum number of interventions were required to achieve maximum success.

Although it is unclear why particular strategies were chosen by studies' authors, it was feasible to categorise the type of intervention strategies used using the $\mathrm{BCW}$ theoretical framework and to assign interventions to the categories of education, persuasion, training, environmental restructuring and enablement. This is the only systematic review and meta-analysis that has evaluated 
the effect of implementation interventions aimed at increasing rates of IVT for AIS using a theoretical framework. However, it should be noted that the studies analysed often did not provide enough detail to unambiguously classify the intervention components based on $\mathrm{BCW}$ intervention functions. The inability to find differences in the effect of different BCW intervention functions in improving IVT rates may be due to the fact that the 5 categories are relatively coarse. The $\mathrm{BCW}$ intervention functions do lend themselves to finer classification but a lack of detailed information in many of the studies reviewed precluded us using this finer-grained classification. Our study also failed to identify any relationship between the number of intervention components and effectiveness of the intervention. A systematic review on multi-component healthcare interventions indicated that multi-component interventions are difficult to implement and seldom implemented as planned [95]. Complex multi-level multi-component interventions or strategies are more difficult to implement and reproduce in the practical setting with fidelity. Multiple strategies may be needed to engage the variety of professionals involved in care such as IVT, which can increase the level of difficulty when implementing or replicating such interventions [96]. Several studies have showed that poor implementation can reduce intervention impact [97]. Therefore, considering the level of complexity in the implementation process, using multi-component $\mathrm{BCW}$ interventions in one package may be challenging.

This study has several strengths and limitations. One limitation, as mentioned above, was that the coding for the $\mathrm{BCW}$ intervention functions was based on the information available in the studies' publications only. Given that the reviewed literature focused on reporting outcome-related aspects of methodology including outcome measures and sample size rather than describing intervention development and content, the literature provides only a very limited understanding of the types of behaviour change interventions that can be effective. A more interdisciplinary approach to designing these trials may be needed to progress the field. If more explicit descriptions of the intervention used were to be published, this may allow for more in-depth classification and, in turn, more capacity to isolate specific $\mathrm{BCW}$ strategies associated with change in AIS practice. It must be acknowledged however, that there is a tension between multi-component and single-focus studies in implementation science. Successful implementation of complex evidence-based practices in any given healthcare setting may require a variety of behaviour change interventions to be implemented. Therefore, evaluating the effectiveness of one $\mathrm{BCW}$ intervention function over another might not always be enough to understand how to effect change on a multi-level multi-component process within a complex health system. One strength of the review is the large number of studies which were included and the total of 40,614 IVT cases, thus giving weight to the results. Other strengths include the rigorous review process used to identify studies and extract data, and the use of the BCW theoretical framework for pooling the intervention effects. Our results may assist researchers with the development of future interventions, including avoiding the assumption that using multiple strategies will necessarily increase intervention effectiveness.

In terms of implications for practice in stroke care, this review suggests that despite the complexity of the IVT care pathway, successfully increasing IVT rates does not necessarily require a complex suite of implementation intervention components, nor should it focus on one specific type of intervention function. Therefore, it seems reasonable to suggest that successful interventions will be those that draw on the diversity of potential interventions to address identified challenges in the local context by paying close attention to each aspect of the patient pathway.

\section{Conclusion}

The evidence we provide does not support using one specific type of BCW intervention strategy over another in the setting of IVT implementation and also that using multiple $\mathrm{BCW}$ intervention strategies in the same programme may not necessarily increase intervention effectiveness. A more inter-disciplinary approach to study design may be needed. A caveat, however, is that the sub-group analysis with RCTs suggested more effect with persuasion, environmental restructuring and enablement approaches. Our results suggest it may be more relevant for policy makers and implementation scientists to select the approaches that best address the major obstacles in a given context. However, because of the high degree of heterogeneity and publication bias, these conclusions cannot be considered robust.

\section{Supplementary Information}

Supplementary information accompanies this paper at https://doi.org/10. 1186/s13012-020-01054-3.

\section{Additional file 1. \\ Additional file 2. \\ Additional file 3. \\ Additional file 4 \\ Additional file 5 . \\ Additional file 6 . \\ Additional file 7 . \\ Additional file 8. \\ Additional file 9.}

Additional file 10.

Abbreviations

AIS: Acute ischemic stroke; BCW: Behaviour change wheel; $\mathrm{Cl}$ : Confidence interval; IVT: Intravenous thrombolysis; OR: Odds ratio; PRISMA: Preferred Reporting Items for Systematic reviews and Meta-analyses 


\section{Acknowledgements}

Not applicable.

\section{Authors' contributions}

MGH directed the review; conducted the screening, data extraction and quality appraisal, carried out the meta-analysis and drafted the manuscript under the supervision of JRA, IJH, CRL and CLP. JRA contributed to the planning of the review, supervised the analysis and commented on the manuscript. SA conducted the screening, data extraction and quality appraisal and commented on the manuscript. TR conducted the screening, data extraction and quality appraisal and commented on the manuscript. AH conducted the meta-analysis and commented on the manuscript. IJH and CRL contributed to the planning of the review and commented on the manuscript. CLP contributed to planning the review, advised throughout the review process and contributed to and commented on the manuscript. All authors read and approved the final manuscript.

\section{Funding}

Not applicable.

\section{Availability of data and materials}

The datasets used and/or analysed during the current study are available from the corresponding author on reasonable request.

\section{Ethics approval and consent to participate}

Not applicable.

\section{Consent for publication}

Not applicable.

\section{Competing interests}

The authors declare that they have no competing interests.

\section{Author details}

${ }^{1}$ School of Medicine and Public Health (SMPH), University of Newcastle (UoN), Callaghan, New South Wales, Australia. ${ }^{2}$ Hunter Medical Research Institute (HMRI), New Lambton Heights, New South Wales, Australia. ${ }^{3} J o h n$ Hunter Hospital, New Lambton Heights, New South Wales, Australia. ${ }^{4}$ Department of Anthropology, Jagannath University, Dhaka, Bangladesh. ${ }^{5}$ Centre for Development, Economics and Sustainability, Monash University, Melbourne, Victoria, Australia. ${ }^{6}$ The Sydney Partnership for Health, Education, Research \& Enterprise (SPHERE), Liverpool, New South Wales, Australia.

Received: 20 January 2020 Accepted: 15 October 2020

Published online: 04 November 2020

\section{References}

1. GBD 2016 Stroke Collaborators. Global, regional, and national burden of stroke, 1990-2016: a systematic analysis for the Global Burden of Disease Study 2016. Lancet Neurol. 2019;18(5):439-58.

2. Bansal S, Sangha KS, Khatri P. Drug treatment of acute ischemic stroke. Am J Cardiovasc Drugs. 2013;13(1):57-69.

3. National Institute of Neurological Disorders and Stroke rt-PA Stroke Study Group. Tissue plasminogen activator for acute ischemic stroke. N Engl J Med. 1995;333(24):1581-7.

4. McDermott M, Skolarus LE, Burke JF. A systematic review and meta-analysis of interventions to increase stroke thrombolysis. BMC Neurol. 2019;19(1):86.

5. Evenson KR, Foraker RE, Morris DL, Rosamond WD. A comprehensive review of prehospital and in-hospital delay times in acute stroke care. Int J Stroke. 2009:4(3):187-99.

6. Eissa A, Krass I, Bajorek BV. Barriers to the utilization of thrombolysis for acute ischaemic stroke. J Clin Pharm Ther. 2012;37(4):399-409.

7. Huang Q, Zhang JZ, Xu WD, Wu J. Generalization of the right acute stroke promotive strategies in reducing delays of intravenous thrombolysis for acute ischemic stroke: a meta-analysis. Medicine (Baltimore). 2018;97(25): e11205

8. Paul CL, Ryan A, Rose S, Attia JR, Kerr E, Koller C, et al. How can we improve stroke thrombolysis rates? A review of health system factors and approaches associated with thrombolysis administration rates in acute stroke care. Implement Sci. 2016;11:51.
9. Campbell M, Fitzpatrick R, Haines A, Kinmonth AL, Sandercock P, Spiegelhalter $\mathrm{D}$, et al. Framework for design and evaluation of complex interventions to improve health. BMJ. 2000;321:694-6.

10. Michie S, Atkins L, West R. The behaviour change wheel-a guide to designing interventions. Great Britain: Silverback; 2014.

11. Craig P, Dieppe P, Macintyre S, Michie S, Nazareth I, Petticrew M; Medical Research Council Guidance. Developing and evaluating complex interventions: the new Medical Research Council guidance. BMJ. 2008;337: a1655.

12. Michie S, van Stralen MM, West R. The behaviour change wheel: a new method for characterising and designing behaviour change interventions. Implement Sci. 2011;6:42.

13. Nilsen P. Making sense of implementation theories, models and frameworks. Implement Sci. 2015;10:53.

14. Shrier I, Boivin JF, Steele RJ, Platt RW, Furlan A, Kakuma R, et al. Should meta-analyses of interventions include observational studies in addition to randomized controlled trials? A critical examination of underlying principles. Am J Epidemiol. 2007;166(10):1203-9.

15. Moher D, Liberati A, Tetzlaff J, Altman DG. Preferred Reporting Items for Systematic Reviews and Meta-Analyses: the PRISMA Statement. J Clin Epidemiol. 2009;62:1006-12.

16. Adams HP Jr, Brott TG, Furlan AJ, Gomez CR, Grotta J, Helgason CM, et al. Guidelines for thrombolytic therapy for acute stroke: a supplement to the guidelines for the management of patients with acute ischemic stroke: a statement for healthcare professionals from a special writing group of the stroke council. Am Heart Assoc Circ. 1996;94(5):1167-74.

17. Kmet L, Lee R, Cook L. Standard quality assessment criteria for evaluating primary research papers from a variety of fields, 2004. Edmonton: Alberta Heritage Foundation for Medical Research; 2011.

18. Akter S, Davies K, Rich JL, Inder KJ. Indigenous women's access to maternal healthcare services in lower- and middle-income countries: a systematic integrative review. Int J Public Health. 2019;64(3):343-53.

19. Higgins JPT, Thomas J, Chandler J, Cumpston M, Li T, Page MJ, Welch VA, editors. Cochrane Handbook for Systematic Reviews of Interventions. 2nd ed. Chichester (UK): John Wiley \& Sons; 2019.

20. Behrens S, Daffertshofer M, Interthal C, Ellinger K, Van Ackern K, Hennerici M. Improvement in stroke quality management by an educational programme. Cerebrovasc Dis. 2002;13:262-6.

21. Morgenstern LB, Bartholomew LK, Grotta JC, Staub L, King M, Chan W. Sustained benefit of a community and professional intervention to increase acute stroke therapy. Arch Intern Med. 2003;163:2198-202.

22. Lattimore SU, Chalela J, Davis L, DeGraba T, Ezzeddine M, Haymore J, et al. Impact of establishing a primary stroke center at a community hospital on the use of thrombolytic therapy: the NINDS Suburban Hospital Stroke Center experience. Stroke. 2003:34:e55-7.

23. Belvis R, Cocho D, Marti-Fabregas J, Pagonabarraga J, Aleu A, Garcia-Bergo $M D$, et al. Benefits of a prehospital stroke code system. Feasibility and efficacy in the first year of clinical practice in Barcelona, Spain. Cerebrovasc Dis. 2005:19:96-101.

24. Wojner-Alexandrov AW, Alexandrov AV, Rodriguez D, Persse D, Grotta JC. Houston paramedic and emergency stroke treatment and outcomes study (HoPSTO). Stroke. 2005;36:1512-8.

25. Ickenstein GW, Horn M, Schenkel J, Vatankhah B, Bogdahn U, Haberl R, et al. The use of telemedicine in combination with a new stroke-code-box significantly increases t-PA use in rural communities. Neurocrit Care. 2005:3(1):27-32.

26. Nam HS, Han SW, Ahn SH, Lee JY, Choi HY, Park IC, et al. Improved time intervals by implementation of computerized physician order entry-based stroke team approach. Cerebrovasc Dis. 2007;23(4):289-93.

27. Demaerschalk BM, Bobrow BJ, Paulsen M. Development of a metropolitan matrix of primary stroke centers: the Phoenix experience. Stroke. 2008;39: 1246-53.

28. Abdullah AR, Smith EE, Biddinger PD, Kalenderian D, Schwamm LH. Advance hospital notification by EMS in acute stroke is associated with shorter door-tocomputed tomography time and increased likelihood of administration of tissue-plasminogen activator. Prehosp Emerg Care. 2008;12:426-31.

29. Quain DA, Parsons MW, Loudfoot AR, Spratt NJ, Evans MK, Russell ML, et al. Improving access to acute stroke therapies: a controlled trial of organised pre-hospital and emergency care. Med J Aust. 2008;189:429-33.

30. Gladstone DJ, Rodan LH, Sahlas DJ, Lee L, Murray BJ, Ween JE, et al. A citywide prehospital protocol increases access to stroke thrombolysis in Toronto. Stroke. 2009:40:3841-4. 
31. Pedragosa A, Alvarez-Sabin J, Molina CA, Sanclemente C, Martín MC, Alonso $F$, et al. Impact of a telemedicine system on acute stroke care in a community hospital. J Telemed Telecare. 2009;15:260-3.

32. Chenkin J, Gladstone DJ, Verbeek PR, Lindsay P, Fang J, Black SE, et al. Predictive value of the Ontario prehospital stroke screening tool for the identification of patients with acute stroke. Prehosp Emerg Care. 2009;13: 153-9.

33. Muller-Nordhorn J, Wegscheider $\mathrm{K}$, Nolte $\mathrm{CH}$, Jungehülsing GJ, Rossnagel $\mathrm{K}$, Reich A, et al. Population-based intervention to reduce prehospital delays in patients with cerebrovascular events. Arch Intern Med. 2009;169:1484-90.

34. Kim SK, Lee SY, Bae HJ, Lee YS, Kim SY, Kang MJ, et al. Pre-hospital notification reduced the door-to-needle time for iv t-PA in acute ischaemic stroke. Eur J Neurol. 2009;16:1331-5

35. Heo JH, Kim YD, Nam HS, Hong KS, Ahn SH, Cho HJ, et al. A computerized in-hospital alert system for thrombolysis in acute stroke. Stroke. 2010;41: 1978-83.

36. Dharmasaroja PA, Muengtaweepongsa S, Kommarkg U. Implementation of Telemedicine and Stroke Network in thrombolytic administration: comparison between walk-in and referred patients. Neurocrit Care. 2010;13:62-6.

37. Bae HJ, Kim DH, Yoo NT, Choi JH, Huh JT, Cha JK, et al. Prehospital notification from the emergency medical service reduces the transfer and intra-hospital processing times for acute stroke patients. J Clin Neurol. 2010; 6:138-42.

38. Sung SF, Huang YC, Ong CT, Chen YW. A parallel thrombolysis protocol with nurse practitioners as coordinators minimized door-to-needle time for acute ischemic stroke. Stroke Res Treat. 2011;2011:198518.

39. Reiner-Deitemyer V, Teuschl Y, Matz K, Reiter M, Eckhardt R, Seyfang L, et al. Helicopter transport of stroke patients and its influence on thrombolysis rates: data from the Austrian Stroke Unit Registry. Stroke. 2011;42:1295-300.

40. Hoegerl C, Goldstein FJ, Sartorius J. Implementation of a stroke alert protocol in the emergency department: a pilot study. J Am Osteopath Assoc. 2011:111:21-7.

41. Etgen T, Freudenberger T, Schwahn M, Rieder G, Sander D. Multimodal strategy in the successful implementation of a stroke unit in a community hospital. Acta Neurol Scand. 2011;123:390-5.

42. Walter S, Kostopoulos P, Haass A, Lesmeister M, Grasu M, Grunwald I, et al. Point-of-care laboratory halves door-to-therapy-decision time in acute stroke. Ann Neurol. 2011;69:581-6.

43. Dirks M, Niessen LW, van Wijngaarden JD, van Wijngaarden JD, Koudstaal PJ, Franke $\mathrm{CL}$, et al. Promoting thrombolysis in acute ischemic stroke. Stroke. 2011;42:1325-30.

44. Addo J, Ayis S, Leon J, Rudd AG, McKevitt C, Wolfe CD. Delay in presentation after an acute stroke in a multiethnic population in South London: the South London stroke register. J Am Heart Assoc. 2012;1:e1685.

45. O'Brien W, Crimmins D, Donaldson W, Risti R, Clarke TA, Whyte S, et al. FAST ER (Face, Arm, Speech, Time, Emergency Response): experience of Central Coast Stroke Services implementation of a pre-hospital notification system for expedient management of acute stroke. J Clin Neurosci. 2012;19:241-5.

46. Berglund A, Svensson L, Sjostrand C, von Arbin M, von Euler M, Wahlgren N, et al. Higher prehospital priority level of stroke improves thrombolysis frequency and time to stroke unit: the Hyper Acute STroke Alarm (HASTA) study. Stroke. 2012;43:2666-70.

47. Prabhakaran S, O'Neill K, Stein-Spencer L, Walter J, Alberts MJ. Prehospital triage to primary stroke centers and rate of stroke thrombolysis. JAMA Neurol. 2013;70:1126-32

48. Bhatt A, Shatila A. Neurohospitalists improve door-to-needle times for patients with ischemic stroke receiving intravenous tPA. Neurohospitalist. 2012;2:119-22.

49. Scott PA, Meurer WJ, Frederiksen SM, Kalbfleisch JD, Xu Z, Haan MN, et al. A multilevel intervention to increase community hospital use of alteplase for acute stroke (INSTINCT): a cluster-randomised controlled trial. Lancet Neurol. 2013;12:139-48.

50. Meretoja A, Weir L, Ugalde M, Yassi N, Yan B, Hand P, et al. Helsinki model cut stroke thrombolysis delays to 25 minutes in Melbourne in only 4 months. Neurology. 2013;81:1071-6.

51. McKinney JS, Mylavarapu K, Lane J, Roberts V, Ohman-Strickland P, Merlin MA. Hospital prenotification of stroke patients by emergency medical services improves stroke time targets. J Stroke Cerebrovasc Dis. 2013;22(2):113-8.

52. Amorim E, Shih MM, Koehler SA, Massaro LL, Zaidi SF, Jumaa MA, et al. Impact of telemedicine implementation in thrombolytic use for acute ischemic stroke: the University of Pittsburgh Medical Center telestroke network experience. J Stroke Cerebrovasc Dis. 2013;22:527-31.
53. Nolte CH, Malzahn U, Kuhnle Y, Ploner CJ, Müller-Nordhorn J, Möckel M. Improvement of door-to-imaging time in acute stroke patients by implementation of an all-points alarm. J Stroke Cerebrovasc Dis. 2013;22: 149-53.

54. Van Schaik SM, Van der Veen B, Van den Berg-Vos RM, Weinstein HC, Bosboom WM, et al. Achieving a door-to-needle time of 25 minutes in thrombolysis for acute ischemic stroke: a quality improvement project. J Stroke Cerebrovasc Dis. 2014;23:2900-6.

55. Fonarow GC, Zhao X, Smith EE, Saver JL, Reeves MJ, Bhatt DL, et al. Door-toneedle times for tissue plasminogen activator administration and clinical outcomes in acute ischemic stroke before and after a quality improvement initiative. JAMA. 2014;311:1632-40.

56. Ebinger $M$, Winter $B$, Wendt $M$, Weber JE, Waldschmidt C, Rozanski M, et al. Effect of the use of ambulance-based thrombolysis on time to thrombolysis in acute ischemic stroke: a randomized clinical trial. JAMA. 2014;311:1622-31

57. Minnerup J, Wersching H, Unrath M, Berger K. Effects of emergency medical service transport on acute stroke care. Eur J Neurol. 2014;21(10):1344-7.

58. Camerlingo M, D'Asero S, Perego L, Rovaris C, Tognozzi M, Moschini L, et al. How to improve access to appropriate therapy and outcome of the acute ischemic stroke: a 24-month survey of a specific pre-hospital planning in Northern Italy. Neurol Sci. 2014;35(9):1359-63.

59. Ruff IM, Ali SF, Goldstein JN, Lev M, Copen WA, McIntyre J, et al. Improving door-to-needle times: a single center validation of the target stroke hypothesis. Stroke. 2014;45(2):504-8.

60. Hesselfeldt R, Gyllenborg J, Steinmetz J, Do HQ, Hejselbæk J, Rasmussen LS. Is air transport of stroke patients faster than ground transport? A prospective controlled observational study. Emerg Med J. 2014;31:268-72.

61. Chen CH, Tang SC, Tsai LK, Hsieh MJ, Yeh SJ, Huang KY, et al. Stroke code improves intravenous thrombolysis administration in acute ischemic stroke. PLoS One. 2014;9:e104862.

62. Martinez-Sanchez P, Miralles A, Sanz DBR, Prefasi D, Sanz-Cuesta BE, Fuentes $B$, et al. The effect of telestroke systems among neighboring hospitals: more and better? The Madrid Telestroke Project. J Neurol. 2014;261:1768-73.

63. Ragoschke-Schumm A, Yilmaz U, Kostopoulos P, Lesmeister M, Manitz M, Walter S, et al. 'Stroke room': diagnosis and treatment at a single location for rapid intraarterial stroke treatment. Cerebrovasc Dis. 2015;40:251-7.

64. Willeit J, Geley T, Schoch J, Rinner H, Tür A, Kreuzer H, et al. Thrombolysis and clinical outcome in patients with stroke after implementation of the Tyrol Stroke Pathway: a retrospective observational study. Lancet Neurol. 2015;14:48-56.

65. Kim A, Lee JS, Kim JE, Paek YM, Chung K, Park JH, et al. Trends in yield of a code stroke program for enhancing thrombolysis. J Clin Neurosci. 2015;22: 73-8.

66. Bladin CF, Molocijz N, Ermel S, Bagot KL, Kilkenny M, Vu M, et al. Victorian Stroke Telemedicine Project: implementation of a new model of translational stroke care for Australia. Intern Med J. 2015:45:951-6.

67. Moran JL, Nakagawa K, Asai SM, Koenig MA. 24/7 neurocritical care nurse practitioner coverage reduced door-to-needle time in stroke patients treated with tissue plasminogen activator. J Stroke Cerebrovasc Dis. 2016;25:1148-52.

68. Busby L, Owada K, Dhungana S, Zimmermann S, Coppola V, Ruban R, et al. CODE FAST: a quality improvement initiative to reduce door-to-needle times. J Neurointerv Surg. 2016;8:661-4.

69. Choi HY, Kim EH, Yoo J, Lee K, Song D, Kim YD, et al. Decision-making support using a standardized script and visual decision aid to reduce doorto-needle time in stroke. J Stroke. 2016;18:239-41.

70. Nishijima H, Ueno $T$, Kon $T$, Haga R, Funamizu $Y$, Arai A, et al. Effects of educational television commercial on pre-hospital delay in patients with ischemic stroke wore off after the end of the campaign. J Neurol Sci. 2017; 381:117-8.

71. Kim DH, Nah HW, Park HS, Choi JH, Kang MJ, Huh JT, et al. Impact of prehospital intervention on delay time to thrombolytic therapy in a stroke center with a systemized stroke code program. J Stroke Cerebrovasc Dis. 2016;25:1665-70.

72. Prabhakaran S, Lee J, O'Neill K. Regional learning collaboratives produce rapid and sustainable improvements in stroke thrombolysis times. Circ Cardiovasc Qual Outcomes. 2016;9(5):585-92.

73. Hubert GJ, Meretoja A, Audebert HJ, Tatlisumak T, Zeman F, Boy S, et al. Stroke thrombolysis in a centralized and a decentralized system (Helsinki and Telemedical Project for Integrative Stroke Care Network). Stroke. 2016; 47:2999-3004 
74. Itrat A, Taqui A, Cerejo R, Briggs F, Cho SM, Organek N, et al. Telemedicine in prehospital stroke evaluation and thrombolysis: taking stroke treatment to the doorstep. JAMA Neurol. 2016;73:162-8.

75. Denti L, Caminiti C, Scoditti U, Zini A, Malferrari G, Zedde ML, et al. Impact on prehospital delay of a stroke preparedness campaign: a SW-RCT (Stepped-Wedge Cluster Randomized Controlled Trial). Stroke. 2017;48(12): 3316-22.

76. Zaidi SF, Shawver J, Espinosa Morales A, Salahuddin H, Tietjen G, Lindstrom $D$, et al. Stroke care: initial data from a county-based bypass protocol for patients with acute stroke. J Neurointerv Surg. 2017;9(7):631-5.

77. Heikkila I, Kuusisto H, Stolberg A, Pakomaki A. Stroke thrombolysis given by emergency physicians cuts in-hospital delays significantly immediately after implementing a new treatment protocol. Scand J Trauma Resusc Emerg Med. 2016;24:46.

78. Hsiao CL, Su YC, Yang FY, Liu CY, Chiang HL, Chen GC, et al. Impact of code stroke on thrombolytic therapy in patients with acute ischemic stroke at a secondary referral hospital in Taiwan. J Chin Med Assoc. 2018;81(11):942-8.

79. Carvalho VS Jr, Picanço MR, Volschan A, Bezerra DC. Impact of simulation training on a telestroke network. Int J Stroke. 2019;14(5):500-7.

80. Gurav SK, Zirpe KG, Wadia RS, Naniwadekar A, Pote PU, Tungenwar A, et al. Impact of "Stroke Code"-Rapid Response Team: an attempt to improve intravenous thrombolysis rate and to shorten door-to-needle time in acute ischemic stroke. Indian J Crit Care Med. 2018;22(4):243-8.

81. Tan BYQ, Ngiam NJH, Sunny S, Kong WY, Tam H, Sim TB, et al. Improvement in door-to-needle time in patients with acute ischemic stroke via a simple stroke activation protocol. J Stroke Cerebrovasc Dis. 2018;27(6):1539-45.

82. Haesebaert J, Nighoghossian N, Mercier C, Termoz A, Porthault S, Derex L, et al. Improving access to thrombolysis and inhospital management times in ischemic stroke: a stepped-wedge randomized trial. Stroke. 2018;49(2): 405-11.

83. Nguyen-Huynh MN, Klingman JG, Avins AL, Rao VA, Eaton A, Bhopale S, et al. Novel Telestroke program improves thrombolysis for acute stroke across 21 hospitals of an integrated healthcare system. Stroke. 2018;49(1): $133-9$.

84. Cone DC, Cooley C, Ferguson J, Harrell AJ, Luk JH, Martin-Gill C, et al. Observational multicenter study of a direct-to-CT protocol for EMS-transported patients with suspected stroke. Prehosp Emerg Care. 2018;22(1):1-6.

85. Sadeghi-Hokmabadi E, Farhoudi M, Taheraghdam A, Rikhtegar R, Ghafouri RR, Asadi R, et al. Prehospital notification can effectively reduce in-hospital delay for thrombolysis in acute stroke. Future Neurol. 2018;13(1):5-11.

86. Meyer BC, Raman R, Hemmen T, Obler R, Zivin JA, Rao R, et al. Efficacy of site-independent telemedicine in the STRokE DOC trial: a randomised, blinded, prospective study. Lancet Neurol. 2008;7(9):787-95.

87. De Luca A, Toni D, Lauria L, Sacchetti ML, Giorgi Rossi P, Ferri M, et al. An emergency clinical pathway for stroke patients--results of a cluster randomised trial (isrctn41456865). BMC Health Serv Res. 2009;9:14

88. Müller-Barna P, Hubert GJ, Boy S, Bogdahn U, Wiedmann S, Heuschmann PU, et al. TeleStroke units serving as a model of care in rural areas: 10-year experience of the TeleMedical project for integrative stroke care. Stroke. 2014;45(9):2739-44.

89. Vidale S, Arnaboldi M, Bezzi G, Bono G, Grampa G, Guidotti M, et al. Reducing time delays in the management of ischemic stroke patients in Northern Italy. Int J Cardiol. 2016;215:431-4.

90. Nardetto L, Dario C, Tonello S, Brunelli MC, Lisiero M, et al. A one-to-one telestroke network: the first Italian study of a web-based telemedicine system for thrombolysis delivery and patient monitoring. Neurol Sci. 2016; 37(5):725-30.

91. Hsieh MJ, Tang SC, Chiang WC, Tsai LK, Jeng JS, Ma MH, et al. Effect of prehospital notification on acute stroke care: a multicenter study. Scand J Trauma Resusc Emerg Med. 2016;24:57.

92. Zhou Y, Xu Z, Liao J, Feng F, Men L, Xu L, et al. New standardized nursing cooperation workflow to reduce stroke thrombolysis delays in patients with acute ischemic stroke. Neuropsychiatr Dis Treat. 2017;13:1215-20.

93. Jeon SB, Ryoo SM, Lee DH, Kwon SU, Jang S, Lee EJ, et al. Multidisciplinary approach to decrease in-hospital delay for stroke thrombolysis. J Stroke. 2017;19(2):196-204.

94. Henry-Morrow TK, Nelson BD, Conahan E, Mathiesen C, Glenn-Porter B, Niehaus MT, et al. An educational intervention allows for greater prehospital recognition of acute stroke. Am J Emerg Med. 2017;35:1959-61.

95. Guise JM, Chang C, Viswanathan M, et al. Systematic Reviews of Complex Multicomponent Health Care Interventions [Internet]. Rockville (MD):
Agency for Healthcare Research and Quality (US); 2014. Available from: https://www.ncbi.nlm.nih.gov/books/NBK194846/.

96. Komro KA, Flay BR, Biglan A, Wagenaar AC. Research design issues for evaluating complex multicomponent interventions in neighborhoods and communities. Transl Behav Med. 2016;6(1):153-9.

97. Durlak JA, DuPre EP. Implementation matters: a review of research on the influence of implementation on program outcomes and the factors affecting implementation. Am J Community Psychol. 2008;41(3-4):327-50.

\section{Publisher's Note}

Springer Nature remains neutral with regard to jurisdictional claims in published maps and institutional affiliations.
Ready to submit your research? Choose BMC and benefit from:

- fast, convenient online submission

- thorough peer review by experienced researchers in your field

- rapid publication on acceptance

- support for research data, including large and complex data types

- gold Open Access which fosters wider collaboration and increased citations

- maximum visibility for your research: over $100 \mathrm{M}$ website views per year

At BMC, research is always in progress.

Learn more biomedcentral.com/submissions 